TRANSACTIONS OF THE

AMERICAN MATHEMATICAL SOCIETY

Volume 364, Number 11, November 2012, Pages 5829-5856

S 0002-9947(2012)05537-1

Article electronically published on June 5, 2012

\title{
ASYMPTOTIC PROPERTIES OF THE QUANTUM REPRESENTATIONS OF THE MODULAR GROUP
}

\author{
LAURENT CHARLES
}

\begin{abstract}
We study the asymptotic behaviour of the quantum representations of the modular group in the large level limit. We prove that each element of the modular group acts as a Fourier integral operator. This provides a link between the classical and quantum Chern-Simons theories for the torus. From this result we deduce the known asymptotic expansion of the Witten-Reshetikhin-Turaev invariants of the torus bundles with hyperbolic monodromy.
\end{abstract}

Quantum Chern-Simons theory was introduced twenty years ago by Witten 13 and Reshetikhin-Turaev [11. It provides among other things invariants of threedimensional manifolds and representations of the mapping class group of surfaces; cf. [1, 12] for an exposition of the theory and [5] for a survey on recent developments. This theory has a semi-classical limit, where the level, an integral parameter denoted by $k$, plays the role of the inverse of the Planck constant. In this paper, we are concerned with the torus and its mapping class group, $\mathrm{Sl}(2, \mathbb{Z})$. We study the large $k$ behaviour of the quantum representation of the modular group.

The quantum representations may be equivalently defined with algebraic or geometrical methods. Geometrically, we consider a line bundle, called the Chern-Simon bundle, over the moduli space of flat $G$-principal bundles on the torus. Here $G$ is a compact Lie group that we assume to be simple and simply connected. Then the modular group acts linearly on the space of holomorphic sections of the $k$-th tensor power of the Chern-Simons bundle. In the the torus case, this construction relies on the modular transformation properties of theta functions (cf. [6] [ 8], 10]).

A part of this construction is standard in geometric quantization: to any compact Kähler manifold with an integral fundamental form one associates the space of holomorphic sections of a prequantum bundle. In this general context the usual tools of microlocal analysis have been introduced. In particular, given a prequantum bundle automorphism, we define a class of operators similar to the Fourier integral operators [2], 3] quantizing it. Our main result, Theorem 9.1, says that each element of $\operatorname{Sl}(2, \mathbb{Z})$ acts as a Fourier integral operator on the quantum spaces, the underlying action on the Chern-Simons bundle being defined through gauge theory. This establishes a clear link between the quantum and classical ChernSimons theories.

As a corollary, we can estimate the character of the quantum representations of the hyperbolic elements of $\mathrm{Sl}(2, \mathbb{Z})$. More generally, under a transversality assumption, one proves that the trace of a Fourier integral operator has an asymptotic expansion, which generalizes in some sense the Lefschetz fixed point formula [3].

Received by the editors July 2, 2010 and, in revised form, December 20, 2010.

2010 Mathematics Subject Classification. Primary 57R56, 35S30, 14K25, 58J28, 58J37.

(C)2012 American Mathematical Society 5829

Reverts to public domain 28 years from publication 
The characters of the quantum representation of the modular group are the threedimensional invariants of the torus bundles. In this way we recover the asymptotic expansion proved by Jeffrey [7, whose leading term is given in terms of the ChernSimons invariants and the torsion of some flat bundles over the torus bundle. The proof in [7] is completely different and relies on the reciprocity formula for Gauss sums. Our result is slightly more general since we treat any hyperbolic element with any simple and simply connected group $G$. But, what is more important, we hope that our analytic method will work in other cases. In the companion paper [4], we prove similar results for the mapping class group in genus $\geqslant 2$.

Besides the semi-classical results, we also give a careful construction of the quantum representations, comparing the geometric and algebraic methods. Strictly speaking, we do not have representations of the modular group but only projective representations which lift to genuine representations of the appropriate extension of $\mathrm{Sl}(2, \mathbb{Z})$. The extensions appearing naturally are not the same in the geometric and the algebraic approach.

The paper is organised as follows. In section 1, we state our result about the asymptotic expansion of the trace of the quantum representations. In section 3, we introduce the phase space of the Chern-Simons theory for the torus, its symplectic structure and prequantum bundle. The relation with gauge theory is the content of section 4. In section 5, we introduce complex structures on the phase space and the associated quantum Hilbert spaces. We exhibit basis in terms of theta functions. The modular group acts naturally on the previous data but does not preserve the complex structure. In section [6 we identify the quantum spaces associated to the various complex structures. This leads to the definition of the quantum representations. In section 7 we compare these representations with the ones defined by algebraic methods. The next two sections are devoted to semi-classical results: section 8 on the identification of the quantum spaces and section 9 on the quantum representations. In a first appendix we prove basic facts on theta functions. In a second appendix we list some notation used in the paper.

\section{Characters of the quantum Representations}

Let $G$ be a compact simple and simply connected Lie group. The phase space of the Chern-Simons theory for an oriented surface $\Sigma$ and group $G$ is the moduli space of flat $G$-principal bundles over $\Sigma$. For a torus, this moduli space identifies with the quotient $\mathrm{T}^{2} / W$, where $\mathrm{T}$ is a maximal torus of $G$ and $W$ is the Weyl group acting diagonally. The modular group $\operatorname{Sl}(2, \mathbb{Z})$, being the mapping class group of the torus, acts on this moduli space. More explicitly, since $T=\mathfrak{t} / \Lambda$, with $\mathfrak{t}$ the Lie algebra of $\mathrm{T}$ and $\Lambda$ the integral lattice, we have a bijection $\mathrm{T}^{2} / W \simeq \mathfrak{t}^{2} /\left(\Lambda^{2} \rtimes W\right)$. Identify $\mathfrak{t}^{2}$ with $\mathbb{R}^{2} \otimes \mathfrak{t}$; then an element $A \in \operatorname{Sl}(2, \mathbb{Z})$ acts on the moduli space by sending the class of $x$ to the class of $\left(A \otimes \mathrm{id}_{\mathfrak{t}}\right) . x$.

Applying geometric quantization, we obtain a family of projective representations of the modular group indexed by a positive integer $k$. Since the construction is rather long, we only give in this introduction the representation of the generators

$$
S=\left(\begin{array}{cc}
0 & -1 \\
1 & 0
\end{array}\right), \quad T=\left(\begin{array}{ll}
1 & 1 \\
0 & 1
\end{array}\right)
$$

of the modular group. Let $B$ be the basic inner product of the Lie algebra of $G$. We choose a set of positive roots and denote by $\mathfrak{A} \subset \mathfrak{t}$ the corresponding open 
fundamental Weyl alcove. We identify the weight lattice $\Lambda^{*} \subset \mathfrak{t}^{*}$ with a lattice of $\mathfrak{t}$ via the basic inner product. The $k$-th representation has a particular basis indexed by the set $\mathfrak{A} \cap k^{-1} \Lambda^{*}$. For any $\lambda, \mu \in \mathfrak{A} \cap k^{-1} \Lambda^{*}$, let

$$
t_{\lambda \mu}=\delta_{\lambda, \mu} \exp (i \pi k B(\lambda, \lambda))
$$

and

$$
s_{\lambda \mu}=i^{p} k^{-\frac{n}{2}} \operatorname{Vol}^{-1}(\mathfrak{t} / \Lambda) \sum_{w \in W}(-1)^{\ell(w)} \exp (-2 i \pi k B(\lambda, w(\mu))),
$$

where $n$ is the rank of $G$ and $p$ is the integral part of $n / 2$.

If the rank of $G$ is even, the map sending $S$ and $T$ to the matrices $s_{\lambda \mu}$ and $t_{\lambda \mu}$ extends to a unitary representation $R_{\mathrm{ev}}^{k}$ of the modular group. If the rank of $G$ is odd, we obtain a representation $R_{\text {odd }}^{k}$ of an extension $\mathrm{Mp}(2, \mathbb{Z})$ of the modular group by $\mathbb{Z} / 2 \mathbb{Z}$. Recall that the metaplectic group $\operatorname{Mp}(2, \mathbb{R})$ is the connected two-cover of $\operatorname{Sl}(2, \mathbb{R})$. Then $\operatorname{Mp}(2, \mathbb{Z})$ is defined as the subgroup of the metaplectic group consisting of the elements which project onto the modular group. The representation $R_{\text {odd }}^{k}$ of the elements projecting onto $S$ and $T$ is given by the matrices $\pm s_{\lambda \mu}$ and $\pm t_{\lambda \mu}$.

Theorem 1.1. Assume the rank of $G$ is even. Then for any hyperbolic element $A \in \mathrm{Sl}(2, \mathbb{Z})$, we have

$$
\operatorname{tr} R_{\mathrm{ev}}^{k}(A)=\frac{(-1)^{2 \epsilon p}}{|W|} \sum_{\substack{w \in W, x \in \mathrm{T}^{2} / \\(A \otimes w) \cdot x=x}}(-1)^{\ell(w)} \frac{e^{i k \theta(A \otimes w, x)}}{|\operatorname{det}(\mathrm{id}-A \otimes w)|^{1 / 2}}+O\left(k^{-1}\right),
$$

where

- $\epsilon=0$ if the trace of $A$ is larger than 2 and $\epsilon=1$ otherwise.

- $\theta(A \otimes w, x)=\pi(B(\mu, p)-B(\gamma, q)+B(\gamma, \mu))$ if $x \in \mathrm{T}^{2} \simeq(\mathfrak{t} / \Lambda)^{2}$ is the class of $(p, q) \in \mathfrak{t}^{2}$ and $(\gamma, \mu)=(A \otimes w)(p, q)-(p, q)$.

If the rank of $G$ is odd, let $\tilde{A} \in \mathrm{Mp}(2, \mathbb{R})$ projecting onto a hyperbolic element $A$ of the modular group. Then the same result holds for the trace of $R_{\mathrm{odd}}^{k}(\tilde{A})$ except that the equivalent has to be multiplied by $\exp \left(i \frac{\pi}{2} \operatorname{ind}(\tilde{A})\right)$, where $\operatorname{ind}(\tilde{A}) \in \mathbb{Z}$ modulo $4 \mathbb{Z}$.

It is a general property of topological quantum field theories that the trace of the quantum representation of an element $A$ of the modular group is the invariant of the mapping torus

$$
M_{A}:=\left(\left(\mathbb{R}^{2} / \mathbb{Z}^{2}\right) \times \mathbb{R}\right) /(A y, t) \sim(y, t+1) .
$$

Here we ignore the complications due to the framing of three-dimensional manifolds and the related fact that we only have a projective representation. For any $\left(x_{1}, x_{2}\right) \in \mathrm{T}^{2}$ and $w \in W$ such that $(A \otimes w) \cdot\left(x_{1}, x_{2}\right)=\left(x_{1}, x_{2}\right)$, consider the flat $G$-principal bundle $P_{A} \rightarrow M_{A}$ whose holonomies along the paths $\gamma(s)=[s, 0,0]$, $[0, s, 0]$ and $[0,0, s]$ are, respectively, $x_{1}, x_{2}$ and $w^{-1}$. Then $(2 \pi)^{-1} \theta(A \otimes w, x)$ is the Chern-Simons invariant of $P_{A}$. This is in agreement with the formula obtained by Witten using the Feynman path integral (heuristic) definition of the three-dimensional invariants. We refer the reader to Jeffrey's paper [7] for more details. In particular, the factor $|\operatorname{det}(\mathrm{id}-A \otimes w)|^{-1}$ appears as an integral of the torsion of the adjoint bundles over the moduli space of flat $G$-principal bundles over $M_{A}$. 


\section{LiE GROUP NOTATION}

Let $\mathfrak{g}$ be a compact simple Lie algebra, and $G$ the corresponding compact connected and simply connected Lie group. Choose a maximal torus T of $G$ and denote by $\mathfrak{t}$ its Lie algebra. The integral lattice $\Lambda$ of $\mathfrak{t}$ is defined as the kernel of the exponential map $\mathfrak{t} \rightarrow$ T. Since $G$ is simply connected, $\Lambda$ is the lattice of $\mathfrak{t}$ generated by the coroots $\alpha^{\vee}$ for the (real) roots $\alpha$.

Let the basic inner product $B$ be the unique invariant inner product on $\mathfrak{g}$ such that for each long root $\alpha, B\left(\alpha^{\vee}, \alpha^{\vee}\right)=2$. In the paper, we will use $B$ to identify $\mathfrak{t}$ with $\mathfrak{t}^{*}$. The basic inner product has the important property that it restricts to an integer-valued $\mathbb{Z}$-bilinear form on $\Lambda$ which takes even values on the diagonal.

We fix a set $\Delta_{+}$of positive roots and let $\mathfrak{t}_{+}$be the corresponding positive open Weyl chamber. Let $\alpha_{0}$ be the highest root and $\mathfrak{A}$ be the open fundamental Weyl alcove

$$
\mathfrak{A}:=\left\{\lambda \in \mathfrak{t}_{+} / \alpha_{0}(\lambda)<1\right\} .
$$

We denote by $W$ the Weyl group of $(G, \mathrm{~T})$. Let $\ell: W \rightarrow\{ \pm 1\}$ be the alternating character of $W$.

\section{The SyMPleCtiC DATA}

In this section we endow $\mathrm{T}^{2}$ with a symplectic form $\omega$ and a prequantum bundle $L$, that is, a complex Hermitian line bundle together with a connection of curvature $\frac{1}{i} \omega$. Furthermore, we introduce commuting actions of the Weyl group and the modular group on $L$.

3.1. A prequantum bundle on $\mathfrak{t}^{2}$. Denote by $p$ and $q$ the projections $\mathfrak{t}^{2} \rightarrow \mathfrak{t}$ on the first and second factor, respectively. Let $\omega$ be the symplectic form on $\mathfrak{t}^{2}$ given by

$$
\omega=2 \pi B(d p, d q) .
$$

Consider the trivial complex line bundle $L_{\mathfrak{t}^{2}}$ over $\mathfrak{t}^{2}$ with fiber $\mathbb{C}$ and connection

$$
d+\frac{\pi}{i}(B(p, d q)-B(q, d p))
$$

Its curvature is $\frac{1}{i} \omega$, so it is a prequantum bundle.

3.2. Heisenberg group and reduction to $\mathrm{T}^{2}$. Introduce the (reduced) Heisenberg group $\mathfrak{t}^{2} \times \mathrm{U}(1)$ with multiplication

$$
(x, u) \cdot(y, v)=\left(x+y, u v \exp \left(\frac{i}{2} \omega(x, y)\right)\right) .
$$

The same formula defines an action of the Heisenberg group on $L_{\mathfrak{t}^{2}}=\mathfrak{t}^{2} \times \mathbb{C}$. This action preserves the trivial metric and the connection. The lattice $\Lambda^{2}$ embeds into the Heisenberg group

$$
\Lambda^{2} \rightarrow \mathfrak{t}^{2} \times \mathrm{U}(1), \quad(p, q) \rightarrow(p, q, \exp (i \pi B(p, q))) .
$$

Using that $B$ takes integral values on $\Lambda$, we prove that this map is a group morphism. Hence we get an action of $\Lambda^{2}$ on $L_{\mathfrak{t}^{2}}$ by automorphisms of prequantum bundles.

By quotienting, we obtain a symplectic form on $\mathrm{T}^{2}=\mathfrak{t}^{2} / \Lambda^{2}$ with a prequantum bundle $L:=\mathfrak{t}^{2} \times \mathbb{C} / \Lambda^{2}$ over $\mathrm{T}^{2}$. 
3.3. Weyl group. Consider the diagonal action of the Weyl group $W$ on $\mathfrak{t}^{2}$ and lift this action trivially on the bundle $L_{\mathfrak{t}^{2}}$. Since $W$ acts on $\mathfrak{t}$ by isometries, $W$ acts on $\mathfrak{t}^{2}$ by linear symplectomorphisms and on $L_{\mathfrak{t}^{2}}$ by isomorphisms of prequantum bundles.

The Weyl group preserves the integral lattice $\Lambda$. Consider the semi-direct product $W \rtimes \Lambda^{2}$, where $W$ acts diagonally on $\Lambda^{2}$. It is easily checked that the actions of $\Lambda^{2}$ and $W$ on the prequantum bundle over $\mathfrak{t}^{2}$ generate an action of $W \rtimes \Lambda^{2}$. Then, quotienting by $\Lambda^{2}$, we obtain an action of $W=\left(W \rtimes \Lambda^{2}\right) / \Lambda^{2}$ on $L$. Since the action of the Weyl group on the base $T^{2}$ is not free, we will not consider the orbifold quotient $\mathrm{T}^{2} / W$ and its prequantum bundle.

3.4. Modular group. Let us consider the symplectic action of the modular group $\Gamma=\mathrm{Sl}(2, \mathbb{Z})$ on $\mathfrak{t}^{2}$ given by

$$
A .(p, q)=(a p+b q, c p+d q), \quad A=\left(\begin{array}{cc}
a & b \\
c & d
\end{array}\right) .
$$

The trivial lift to the prequantum bundle $L_{\mathrm{t}^{2}}$ preserves the metric and the connection. Furthermore, this action and the action of $\Lambda^{2}$ define an action of the semi-direct product $\Gamma \rtimes \Lambda^{2}$. To prove this, one has to use that $B(p, q)$ is integral when $p, q \in \Lambda$ and even if furthermore $p=q$. Consequently, we get an action of the modular group on $L$ by prequantum bundle isomorphisms. Observe that the Weyl group action on $L$ commutes with the modular action.

\section{Chern-Simons theory}

We explain how the definitions of the previous section can be deduced from gauge theory. Our aim is only to motivate the constructions. No proof in the paper relies on the gauge theoretic considerations.

The phase space of the Chern-Simons theory for an oriented surface $\Sigma$ is the moduli space of representations of the fundamental group of $\Sigma$ in $G$. When $\Sigma$ is a torus, the fundamental group is the free Abelian group with two generators, so each representation is given by a pair of commuting elements of $G$ unique up to conjugation. In the same way that $G / \operatorname{Ad} G \simeq \mathrm{T} / W$, one shows that these representations are conjugate to a representation in the maximal torus $\mathrm{T}$, uniquely up to the action of the Weyl group. So the moduli space of representations for the torus is $\mathrm{T}^{2} / W$.

4.1. Gauge theory presentation. Consider the space $\Omega^{1}(\Sigma, \mathfrak{g})$ of connections of the trivial $G$-principal bundle with base $\Sigma$. It is a symplectic vector space with symplectic form given by

$$
\Omega(a, b)=2 \pi \int_{\Sigma} B(a, b)
$$

The gauge group $\mathcal{C}^{\infty}(\Sigma, G)$ acts on $\Omega^{1}(\Sigma, \mathfrak{g})$ by symplectic affine isomorphisms:

$$
\text { g.a }=\operatorname{Ad}_{g} a-g^{*} \bar{\theta},
$$

where $\bar{\theta} \in \Omega^{1}(G, \mathfrak{g})$ is the right-invariant Maurer-Cartan form. Each gauge class of flat connections is determined by its holonomy representation. The quotient of the 
space of flat connections by the gauge group may be viewed as a symplectic quotient which defines a symplectic structure on the moduli space of representations.

In the case where $\Sigma$ is a torus, we can avoid this infinite-dimensional quotient by proceeding as follows. Represent $\Sigma$ as the quotient $\mathbb{R}^{2} / \mathbb{Z}^{2}$ with coordinates $x, y$. Then the map

$$
\mathfrak{t}^{2} \rightarrow \Omega^{1}(\Sigma, \mathfrak{g}), \quad(p, q) \rightarrow p d x+q d y
$$

is a symplectic embedding where the symplectic form of $\mathfrak{t}^{2}$ is the one of section 3.1. This embedding is equivariant with respect to the action of $W \rtimes \Lambda^{2}$ on $\mathfrak{t}^{2}$ and the morphism from $W \rtimes \Lambda^{2}$ to the gauge group sending an element $w \in W$ to the constant gauge transform $w$ and $(\dot{p}, \dot{q}) \in \Lambda^{2}$ to $\exp (-(x \dot{p}+y \dot{q}))$. Furthermore, each gauge class of flat connections intersects the image of the embedding.

4.2. Prequantum bundle. Consider the trivial line bundle with base $\Omega^{1}(\Sigma, \mathfrak{g})$ and connection $d+\frac{1}{i} \alpha$, where $\alpha$ is the primitive of $\Omega$ given by

$$
\left.\alpha\right|_{a}(b)=\frac{1}{2} \Omega(a, b) .
$$

This is a prequantum bundle and the gauge group action lifts to it in such a way that it preserves the trivial metric and the connection. Explicitly, the action of $g \in \mathcal{C}^{\infty}(\Sigma, G)$ on $(a, u) \in \Omega^{1}(\Sigma, \mathfrak{g}) \times \mathbb{C}$ is given by

$$
g \cdot(a, u)=\left(g \cdot a, \exp \left(-2 i \pi W(g)-i \pi \int_{\Sigma} B\left(g^{*} \theta, a\right)\right) u\right)
$$

where $\theta \in \Omega^{1}(G, \mathfrak{g})$ is the left-invariant Maurer-Cartan one-form and $W(g)$ is the Wess-Zumino-Witten term

$$
W(g)=\int_{M} \tilde{g}^{*} \chi
$$

Here $M$ is any three-dimensional compact oriented manifold with boundary $\Sigma$, $\tilde{g} \in \mathcal{C}^{\infty}(M, G)$ is any extension of $g$ and $\chi$ is the Cartan three-form defined in terms of the left- or right-invariant Maurer Cartan forms by

$$
\chi=\frac{1}{12} B([\theta, \theta], \theta)=\frac{1}{12} B([\bar{\theta}, \bar{\theta}], \bar{\theta}) .
$$

Since $B$ is the basic inner product, the cohomology class of $\chi$ is integral.

Now assume that $\Sigma$ is a torus and consider the equivariant embedding of $\mathfrak{t}^{2}$ in $\Omega^{1}(\Sigma, \mathfrak{g})$ defined in section 4.1. By pulling back, we obtain a prequantum bundle on $\mathfrak{t}^{2}$ together with an action of $\Lambda^{2} \rtimes W$ on it. It is not difficult to check that this bundle and this action are exactly the ones we introduced in section 3

4.3. Mapping class group. The group of orientation preserving diffeomorphisms of $\Sigma$ acts symplectically on $\Omega^{1}(\Sigma, \mathfrak{g})$. The trivial lift to the prequantum bundle preserves the connection and the trivial metric. After quotienting by the gauge group, this defines an action of the mapping class group on the moduli space of representation and its prequantum bundle.

When $\Sigma$ is a torus, we recover the action of $\Gamma$ introduced in section 3.4 For any $A \in \Gamma$, define the diffeomorphism of the torus

$$
\varphi_{A}(x, y)=(d x-c y,-b x+a y),
$$


where $a, b, c$, and $d$ are the coefficients of $A$. On one hand, we recover the usual formula by considering the basis $\alpha=(0,-1)$ and $\beta=(1,0)$. Indeed, $\varphi_{A}(\alpha)=$ $a \alpha+b \beta$ and $\varphi_{A}(\beta)=c \alpha+d \beta$. On the other hand,

$$
\varphi_{A}^{*}(p d x+q d y)=(a p+b q) d x+(c p+d q) d y
$$

which corresponds to the action of section 3.4

\section{Quantization}

Let us begin with a brief description of the general set-up. Consider a symplectic manifold $(M, \omega)$ with a prequantum bundle $L \rightarrow M$. Assume $(M, \omega)$ is endowed with a compatible positive complex structure, so $\omega$ is a $(1,1)$-form and $-i \omega(Z, \bar{Z})>$ 0 for any nonvanishing tangent vector $Z$ of type $(1,0)$. Then the prequantum bundle has a unique holomorphic structure such that the local holomorphic sections satisfy the Cauchy-Riemann equations:

$$
\nabla_{\bar{Z}} s=0, \quad \text { for any vector field } Z \text { of type }(1,0) \text {. }
$$

The quantum space associated to these data is the space $H^{0}\left(M, L^{k}\right)$ of holomorphic sections of $L^{k}$. It has a natural scalar product obtained by integrating the pointwise scalar product of sections against the Liouville measure $\left|\omega^{n}\right| / n$ !.

In a first subsection we introduce complex structures on $\mathrm{T}^{2}$ and define a basis of the quantum space by using theta functions. We also describe the action of the matrices $S$ and $T$ of $\Gamma$ in this basis. Since our presentation is not usual, we provide proofs in the appendix. In the literature, the equation describing the modular action is called the functional equation. Good references are [8] and [10]. Next we move on to a subspace of equivariant sections with respect to the Weyl group action, the so-called alternating sections. We compute the actions of $S$ and $T$ in this space.

5.1. Complex structure and theta functions. Denote by $\mathcal{H}_{+}$the Poincaré upper half-plane

$$
\mathcal{H}_{+}=\{x+i y / x, y \in \mathbb{R}, y>0\} .
$$

Let $\tau \in \mathcal{H}_{+}$. Identify $\mathfrak{t}^{2}$ with $\mathfrak{t}_{\mathbb{C}}=\mathfrak{t} \otimes \mathbb{C}$ by the isomorphism sending $(p, q)$ to $p+\tau q$. Hence $\mathfrak{t}^{2}$ becomes a complex vector space and $\mathrm{T}^{2}$ inherits a complex structure. One may compute the symplectic form $\omega$ in terms of the complex coordinate $\zeta=p+\tau q$

$$
\omega=\frac{2 \pi}{\bar{\tau}-\tau} B(d \zeta, d \bar{\zeta})
$$

It is a positive real form of type $(1,1)$. So the prequantum bundle $L$ has a unique holomorphic structure compatible with the connection. We denote by $H_{\tau}^{0}\left(\mathrm{~T}^{2}, L^{k}\right)$ its space of holomorphic sections.

Consider the section of $L_{\mathfrak{t}^{2}}$,

$$
s=\exp (i \pi B(\zeta, q))
$$

Its covariant derivative is $2 i \pi B(d \zeta, q) \otimes s$. Since this form is of type $(1,0), s$ is holomorphic. Furthermore, $s$ doesn't vanish anywhere. So the holomorphic sections of $L^{k}$ identify with the sections over $\mathfrak{t}^{2}$ of the form $f s^{k}$ such that $f: \mathfrak{t}^{2} \rightarrow \mathbb{C}$ is holomorphic and $f s^{k}$ is $\Lambda^{2}$-invariant. 
As previously, we embed $\Lambda^{*}$ in $\mathfrak{t}$ via the identification given by the basic inner product. Recall that $\Lambda \subset \Lambda^{*}$. For any $\mu \in k^{-1} \Lambda^{*}$, consider the theta function

$$
\Theta_{\mu, k}(p, q)=\sum_{\gamma \in \mu+\Lambda} \exp \left(2 i \pi k\left(\frac{\tau}{2} B(\gamma, \gamma)-B(\zeta, \gamma)\right)\right) .
$$

This series converges uniformly on compact sets to a holomorphic function; it depends only on $\mu \bmod \Lambda$.

Theorem 5.1. For any integer $k$, the sections $\Theta_{\mu, k} s^{k}$, where $\mu$ runs over $k^{-1} \Lambda^{*}$ $\bmod \Lambda$, are $\Lambda^{2}$-invariant and form an orthonormal basis of $H_{\tau}^{0}\left(\mathrm{~T}^{2}, L^{k}\right)$. Furthermore,

$$
\left\|\Theta_{\mu, k} s^{k}\right\|^{2}=\left(\frac{2 \pi}{k}\right)^{n / 2}\left(\frac{2 i \pi}{\tau-\bar{\tau}}\right)^{n / 2} \operatorname{Vol}(\mathfrak{t} / \Lambda),
$$

where $\operatorname{Vol}(\mathfrak{t} / \Lambda)$ is the Riemannian volume determined by $B$.

Recall that the modular group acts on $\mathrm{T}^{2}$ and its prequantum bundle. The induced action on the sections of $L^{k}$ doesn't preserve the space $H_{\tau}^{0}\left(\mathrm{~T}^{2}, L^{k}\right)$ because of the complex structure. Actually, $A \in \Gamma$ acts as a holomorphic map from $\left(\mathrm{T}^{2}, j_{\tau}\right)$ to $\left(\mathrm{T}^{2}, j_{A \tau}\right)$ with

$$
A \tau=\frac{a \tau-b}{-c \tau+d}
$$

So for any $\tau, A$ acts as an isomorphism

$$
H_{\tau}^{0}\left(\mathrm{~T}^{2}, L^{k}\right) \rightarrow H_{A \tau}^{0}\left(\mathrm{~T}^{2}, L^{k}\right) .
$$

One may compute explicitly this isomorphism on the basis of theta functions when $A$ is the matrix $S$ or $T$. We make explicit the dependence in $\tau$ in our notation to avoid any ambiguity.

Theorem 5.2. For any $\tau \in \mathcal{H}_{+}$and $\mu \in k^{-1} \Lambda^{*}$, one has

$$
S .\left(\Theta_{\mu, k}^{\tau} s_{\tau}^{k}\right)=C \sum_{\mu^{\prime} \in k^{-1} \Lambda^{*} \bmod \Lambda} \exp \left(-2 i \pi k B\left(\mu, \mu^{\prime}\right)\right) \Theta_{\mu^{\prime}, k}^{S . \tau} s_{S . \tau}^{k}
$$

with $C=(S . \tau / i)^{n / 2} k^{-n / 2} \operatorname{Vol}(\mathfrak{t} / \Lambda)^{-1}$ and

$$
T .\left(\Theta_{\mu, k}^{\tau} s_{\tau}^{k}\right)=\exp (i \pi k B(\mu, \mu)) \Theta_{\mu, k}^{T \cdot \tau} s_{T . \tau}^{k} .
$$

Here $(\tau / i)^{n / 2}$ is the determination continuous with respect to $\tau$ and equal to 1 when $\tau=i$.

5.2. Alternating sections. The action of the Weyl group on $\mathrm{T}^{2}$ is holomorphic with respect to the complex structure defined by any $\tau \in \mathcal{H}_{+}$. Let us consider the alternating sections of $L^{k}$, i.e. the sections $\Psi$ satisfying

$$
w \cdot \Psi=(-1)^{\ell(w)} \Psi, \quad \forall w \in W .
$$

For any $\mu \in k^{-1} \Lambda^{*}$, let

$$
\chi_{\mu, k}=\sum_{w \in W}(-1)^{\ell(w)} \Theta_{w(\mu), k} s^{k} .
$$

Recall that we denote by $\mathfrak{A}$ the fundamental open Weyl alcove.

Theorem 5.3. The family $\left(\chi_{\mu, k}, \mu \in \mathfrak{A} \cap k^{-1} \Lambda^{*}\right)$ is a basis of the space of alternating holomorphic sections of $L^{k}$. 
Proof. Using that the Weyl group action preserves $\Lambda$ and $B$, we check that for any $w \in W$,

$$
w \cdot\left(\Theta_{\mu, k} s^{k}\right)=\Theta_{w(\mu), k} s^{k} .
$$

So $\chi_{\mu, k}$ is alternating.

For any root $\alpha$ and integer $n$, the orthogonal reflexion with respect to the hyperplane $\alpha^{-1}(n)$ belongs to the affine Weyl group $W \rtimes \Lambda$. So for any $\mu \in \alpha^{-1}(n)$, there exists $w \in W$ with $\ell(w)=1$ such that $w(\mu)=\mu$ modulo $\Lambda$. Hence $\chi_{\mu, k}=-\chi_{w(\mu), k}=-\chi_{\mu, k}$, so $\chi_{\mu, k}$ vanishes.

Recall that the affine Weyl group $W \rtimes \Lambda$ acts simply transitively on the set of components of $\mathfrak{t} \backslash \bigcup_{\alpha, n} \alpha^{-1}(n)$ and that $\mathfrak{A}$ is one of these components. The result follows from Theorem 5.1

The modular action and the action of the Weyl group on $\mathrm{T}^{2}$ and $L$ commute. So the representation of the modular group preserves the subspace of alternating sections.

Theorem 5.4. For any $\tau \in \mathcal{H}_{+}$and $\mu \in \mathfrak{A} \cap k^{-1} \Lambda^{*}$, one has

$$
S .\left(\chi_{\mu, k}^{\tau} s_{\tau}^{k}\right)=C \sum_{\substack{\mu^{\prime} \in \mathfrak{A} \cap k^{-1} \Lambda^{*}, w \in W}}(-1)^{\ell(w)} \exp \left(-2 i \pi k B\left(\mu, w\left(\mu^{\prime}\right)\right)\right) \chi_{\mu^{\prime}, k}^{S . \tau} s_{S . \tau}^{k},
$$

with $C$ defined as in Theorem 5.2 and

$$
T \cdot\left(\chi_{\mu, k}^{\tau} s_{\tau}^{k}\right)=\exp (i \pi k B(\mu, \mu)) \chi_{\mu, k}^{T \cdot \tau} s_{T \cdot \tau}^{k} .
$$

Proof. The second formula follows from Theorem 5.2 by using that the Weyl group acts isometrically on $\mathfrak{t}$. Let us prove the first one. By Theorem 5.2,

$$
\begin{aligned}
S .\left(\chi_{\mu, k}^{\tau} s_{\tau}^{k}\right) & \left.=C \sum_{\substack{\mu^{\prime} \in k^{-1} \Lambda^{*} \bmod \Lambda \\
w \in W}}(-1)^{\ell(w)} \exp \left(-2 i \pi k B\left(w(\mu), \mu^{\prime}\right)\right)\right) \theta_{\mu^{\prime}, k}^{S . \tau} s_{S . \tau}^{k} \\
& =C \sum_{\substack{\mu^{\prime} \in k^{-1} \Lambda^{*} \bmod \Lambda, w \in W}}(-1)^{\ell(w)} \exp \left(-2 i \pi k B\left(\mu, \mu^{\prime}\right)\right) \theta_{w\left(\mu^{\prime}\right), k}^{S . \tau} s_{S . \tau}^{k} \\
& =C \sum_{\mu^{\prime} \in k^{-1} \Lambda^{*} \bmod \Lambda} \exp \left(-2 i \pi k B\left(\mu, \mu^{\prime}\right)\right) \chi_{\mu^{\prime}, k}^{S . \tau} s_{S . \tau}^{k} \\
& =C \sum_{\substack{\mu^{\prime} \in \mathfrak{A} \cap k^{-1} \Lambda^{*}, w \in W}} \exp \left(-2 i \pi k B\left(\mu, w\left(\mu^{\prime}\right)\right)\right) \chi_{w\left(\mu^{\prime}\right), k}^{S . \tau} s_{S . \tau}^{k} \cdot
\end{aligned}
$$

In the last line, we used that the affine Weyl group acts simply transitively on the set of connected components of $\mathfrak{t} \backslash \bigcup \alpha^{-1}(n)$ and that $\chi_{\mu}$ vanishes if $\mu \in \alpha^{-1}(n)$. Finally,

$$
S .\left(\chi_{\mu, k}^{\tau} s_{\tau}^{k}\right)=C \sum_{\substack{\mu^{\prime} \in \mathfrak{A} \cap k^{-1} \Lambda^{*} \\ w \in W}}(-1)^{\ell(w)} \exp \left(-2 i \pi k B\left(\mu, w\left(\mu^{\prime}\right)\right)\right) \chi_{\mu^{\prime}, k}^{S . \tau} s_{S . \tau}^{k},
$$

since the $\chi_{\mu, k}$ 's are alternating. 


\section{Geometric quantum representation}

We introduce a representation of the modular group on the quantum spaces. To do this we identify the various spaces $H_{\tau}^{0}\left(\mathrm{~T}^{2}, L^{k}\right)$ via the sections $\Theta_{\mu, k}^{\tau} s_{\tau}^{k}$. Unfortunately, the norm of these sections and the action of the modular group depend on $\tau$ as it appears in Theorems 5.1 and 5.2 . We introduce half-form bundles to correct this.

6.1. Half-form bundles. Let us begin with some definitions. Consider a symplectic manifold $M$ with a prequantum bundle $L$ and a positive compatible complex structure. Then a half-form bundle is a complex line bundle $\delta$ over $M$ with an isomorphism from $\delta^{2}$ to the canonical bundle of $M$. A half-form bundle admits a natural metric and a natural holomorphic structure making the isomorphism with the canonical bundle a morphism of Hermitian holomorphic bundle. The quantization of $M$ with metaplectic correction is then the space of holomorphic sections of $L^{k}$ tensored with $\delta$. The scalar product is defined by integrating the pointwise norm of sections against the Liouville measure.

Let us return to our particular situation. Consider $\Omega \in \bigwedge^{n} \mathfrak{t}_{\mathbb{C}}^{*}$ such that for a basis $\left(\gamma_{i}\right)$ of $\Lambda$, one has

$$
\Omega\left(\gamma_{1} \wedge \ldots \wedge \gamma_{n}\right)=1
$$

$\Omega$ is uniquely defined up to a plus or minus sign. For any $\tau \in \mathcal{H}_{+}$, we defined a complex structure $j_{\tau}$ on $\mathrm{T}^{2}$ via the isomorphism

$$
\mathrm{T}^{2} \rightarrow \mathfrak{t}_{\mathbb{C}} /(\Lambda+\tau \Lambda), \quad[p, q] \rightarrow[p+\tau q] .
$$

So the holomorphic tangent bundle of $\left(\mathrm{T}^{2}, j_{\tau}\right)$ is naturally isomorphic to the trivial bundle with fiber $\mathfrak{t}_{\mathbb{C}}$. Consequently the canonical bundle is naturally isomorphic to the trivial bundle with fiber $\bigwedge^{n} \mathfrak{t}_{\mathbb{C}}^{*}$. Denote by $\Omega_{\tau}$ the section of the canonical bundle which is sent into the constant section equal to $\Omega$ by this trivialization.

Lemma 6.1. The section $\Omega_{\tau}$ is holomorphic and has a constant pointwise norm equal to $\left(\frac{\tau-\bar{\tau}}{2 i \pi}\right)^{n / 2} \operatorname{Vol}(\mathfrak{t} / \Lambda)^{-1}$.

Proof. Introduce an orthonormal basis $\left(u_{i}\right)$ of $\mathfrak{t}$ and denote by $\left(p_{i}\right)$ and $\left(q_{i}\right)$ the associated linear coordinates of $\mathfrak{t}^{2}$. Let $\zeta_{\tau}^{i}$ be the complex coordinate $p_{i}+\tau q_{i}$. Then if $\left(\gamma_{i}\right)$ is a basis of $\Lambda$, one has

$$
\begin{aligned}
d \zeta_{\tau}^{1} \wedge \ldots \wedge d \zeta_{\tau}^{n}\left(\gamma_{1}, \ldots, \gamma_{n}\right) & =d p_{1} \wedge \ldots \wedge d p_{n}\left(\gamma_{1}, \ldots, \gamma_{n}\right) \\
& = \pm \operatorname{Vol}(\mathfrak{t} / \Lambda)
\end{aligned}
$$

where the plus or minus sign depends on the orientation of the various basis. Consequently,

$$
\Omega_{\tau}= \pm \operatorname{Vol}(\mathfrak{t} / \Lambda)^{-1} d \zeta_{\tau}^{1} \wedge \ldots \wedge d \zeta_{\tau}^{n}
$$

Now, by definition, the Hermitian product of two tangent vectors $X, Y$ of type $(1,0)$ is given by $\frac{1}{i} \omega(X, \bar{Y})$. Since

$$
\omega=2 \pi \sum d p_{i} \wedge d q_{i}=i \frac{2 i \pi}{\tau-\bar{\tau}} \sum d \zeta_{\tau}^{i} \wedge d \bar{\zeta}_{\tau}^{i}
$$

the vectors $\partial_{\zeta_{\tau}^{i}}$ are mutually orthogonal and

$$
\left|\partial_{\zeta_{\tau}^{i}}\right|^{2}=\frac{2 i \pi}{\tau-\bar{\tau}}
$$


So the $d \zeta_{\tau}^{i}$ are mutually orthogonal and

$$
\left|d \zeta_{\tau}^{i}\right|^{2}=\frac{\tau-\bar{\tau}}{2 i \pi}
$$

which implies

$$
\left|d \zeta_{\tau}^{1} \wedge \ldots \wedge d \zeta_{\tau}^{n}\right|^{2}=\left(\frac{\tau-\bar{\tau}}{2 i \pi}\right)^{n}
$$

and concludes the proof.

Let $\delta$ be a complex vector line and $\varphi$ be an isomorphism $\delta^{\otimes 2} \rightarrow \wedge^{\text {top }} \mathfrak{t}_{\mathbb{C}}^{*}$. Let $\Omega^{1 / 2} \in \delta$ be such that

$$
\varphi\left(\Omega^{1 / 2} \otimes \Omega^{1 / 2}\right)=\Omega .
$$

For any $\tau \in \mathcal{H}_{+}$, the trivial bundle $\delta_{\tau}$ with base $\mathrm{T}^{2}$ and fiber $\delta$ is a half-form bundle, with the squaring map sending $\Omega^{1 / 2}$ into $\Omega_{\tau}$. By the previous lemma, the constant section equal to $\Omega^{1 / 2}$ is a holomorphic section of $\delta_{\tau}$ with constant pointwise norm equal to $\left(\frac{\tau-\bar{\tau}}{2 i \pi}\right)^{n / 4} \operatorname{Vol}(\mathfrak{t} / \Lambda)^{-1 / 2}$.

Instead of sections of $L^{k}$, now consider sections of $L^{k} \otimes \delta_{\tau}$. The multiplication by $\Omega^{1 / 2}$ is an isomorphism

$$
H_{\tau}^{0}\left(\mathrm{~T}^{2}, L^{k}\right) \simeq H_{\tau}^{0}\left(\mathrm{~T}^{2}, L^{k} \otimes \delta_{\tau}\right)
$$

of vector spaces. We deduce from Theorem 5.1 and Lemma 6.1 the following theorem.

Theorem 6.2. For any $\tau \in \mathcal{H}_{+}$, the sections

$$
\Theta_{\mu, k}^{\tau} s_{\tau}^{k} \otimes \Omega^{1 / 2}, \quad \mu \in k^{-1} \Lambda^{*} \bmod \Lambda,
$$

form a basis of $H_{\tau}^{0}\left(\mathrm{~T}^{2}, L^{k} \otimes \delta_{\tau}\right)$. They are mutually orthogonal and

$$
\left\|\Theta_{\mu, k}^{\tau} s_{\tau}^{k} \otimes \Omega^{1 / 2}\right\|^{2}=\left(\frac{2 \pi}{k}\right)^{n / 2}
$$

For any $\tau_{1}$ and $\tau_{2}$ in $\mathcal{H}_{+}$, let $\Psi_{\tau_{1}, \tau_{2}, k}$ be the isomorphism from $H_{\tau_{1}}^{0}\left(\mathrm{~T}^{2}, L^{k} \otimes \delta_{\tau_{1}}\right)$ to $H_{\tau_{2}}^{0}\left(\mathrm{~T}^{2}, L^{k} \otimes \delta_{\tau_{2}}\right)$ defined by

$$
\Psi_{\tau_{1}, \tau_{2}, k}\left(\Theta_{\mu, k}^{\tau_{1}} s_{\tau_{1}}^{k} \otimes \Omega^{1 / 2}\right)=\Theta_{\mu, k}^{\tau_{2}} s_{\tau_{2}}^{k} \otimes \Omega^{1 / 2}, \quad \forall \mu .
$$

By the previous theorem, $\Psi_{\tau_{1}, \tau_{2}, k}$ is a unitary map.

6.2. Modular action. Let $A \in \Gamma$ be the matrix with coefficients $a, b, c$ and $d$. The map $\varphi_{A}(p, q)=(a p+b q, c p+d q)$ is a holomorphic map from $\left(\mathrm{T}^{2}, j_{\tau}\right)$ to $\left(\mathrm{T}^{2}, j_{A \cdot \tau}\right)$. Using the coordinates introduced in the proof of Lemma 6.1, we show that

$$
\varphi_{A}^{*} \Omega_{A . \tau}=(-c \tau+d)^{-n} \Omega_{\tau} .
$$

We will lift the action of $A$ to the half-form bundles in such a way that it squares to $\left(\varphi_{A}^{*}\right)^{-1}$. Since $(-c \tau+d)^{n}$ doesn't admit a preferred square root, we have to pass to an extension of the modular group. 
Let $\Gamma_{2}$ be the set of pairs $(A, e)$ where $A \in \Gamma$ and $e$ is a continuous function from $\mathcal{H}_{+}$to $\mathbb{C}$ satisfying

$$
e(\tau)^{2}=(-c \tau+d)^{n} \quad \text { if } \quad A=\left(\begin{array}{ll}
a & b \\
c & d
\end{array}\right) .
$$

$\Gamma_{2}$ is a group with the product given by $(A, e) \cdot\left(A^{\prime}, e^{\prime}\right)=\left(A A^{\prime}, e^{\prime \prime}\right)$, where $e^{\prime \prime}(\tau)=$ $e\left(A^{\prime} \tau\right) e^{\prime}(\tau) . \Gamma_{2}$ acts on the product $\mathcal{H}_{+} \times \mathrm{T}^{2} \times \delta$

$$
(A, e) \cdot\left(\tau, p, q, z \Omega^{1 / 2}\right)=\left(\frac{a \tau-b}{-c \tau+d}, a p+b q, c p+d q, z e(\tau) \Omega^{1 / 2}\right) .
$$

Restricting to a particular value $\tau$, we obtain a morphism from $\delta_{\tau}$ to $\delta_{A . \tau}$ lifting the action of $A$ on $\mathrm{T}^{2}$. The important point is that the square of this isomorphism is the natural map between the canonical bundles of $\left(\mathrm{T}^{2}, j_{\tau}\right)$ and $\left(\mathrm{T}^{2}, j_{A \tau}\right)$. Since the Hermitian and holomorphic structures of a half-form bundle are determined by the corresponding canonical bundle, the morphism $\delta_{\tau} \rightarrow \delta_{A . \tau}$ that we consider is a unitary holomorphic isomorphism.

As previously we trivially lift the action of $\Gamma$ to the prequantum bundle. Then we obtain for any $(A, e, \tau) \in \Gamma_{2} \times \mathcal{H}_{+}$an isomorphism

$$
\varphi_{(A, e)^{-1}, \tau}^{*}: H_{\tau}^{0}\left(\mathrm{~T}^{2}, L^{k} \otimes \delta_{\tau}\right) \rightarrow H_{A \cdot \tau}^{0}\left(\mathrm{~T}^{2}, L^{k} \otimes \delta_{A . \tau}\right) .
$$

By trivial reason, it is a unitary map. Miraculously, these isomorphisms all fit together.

Proposition 6.3. For any $\tau_{1}, \tau_{2} \in \mathcal{H}_{+}$and any $(A, e) \in \Gamma_{2}$, the diagram

$$
\begin{array}{cr}
H_{\tau_{1}}^{0}\left(\mathrm{~T}^{2}, L^{k} \otimes \delta_{\tau_{1}}\right) & \stackrel{\varphi_{(A, e)-1, \tau}^{*}}{\longrightarrow} H_{A \cdot \tau_{1}}^{0}\left(\mathrm{~T}^{2}, L^{k} \otimes \delta_{A \cdot \tau_{1}}\right) \\
\downarrow \Psi_{\tau_{1}, \tau_{2}} & \\
H_{\tau_{2}}^{0}\left(\mathrm{~T}^{2}, L^{k} \otimes \delta_{\tau_{2}}\right) & \stackrel{\varphi_{\left(A, \tau_{1}, A \cdot \tau_{2}\right.}^{*}, \tau}{\longrightarrow} H_{A \cdot \tau_{2}}^{0}\left(\mathrm{~T}^{2}, L^{k} \otimes \delta_{A \cdot \tau_{2}}\right)
\end{array}
$$

commutes.

Proof. It is sufficient to prove it for $(A, e)=(T, 1),(S, e)$ or (id, -1$)$, since these elements generate $\Gamma$. The actions of $(T, 1)$ and $(S, e)$ in the basis $\Theta_{\mu, k}^{\tau} s_{\tau}^{k} \Omega^{1 / 2}$ are given by the same formulas as in Theorem 5.2 except that the constant $C$ has to be replaced by

$$
C^{\prime}=C e(\tau)=e(i) k^{-n / 2} \operatorname{Vol}(\mathfrak{t} / \Lambda)^{-1} .
$$

This proves the result because $C^{\prime}$ does not depend on $\tau$.

Corollary 6.4. For any $\tau$, the map

$$
R_{2}:(A, e) \rightarrow \varphi_{(A, e)^{-1}, \tau}^{*} \circ \Psi_{\tau, A^{-1} \tau}
$$

is a unitary representation of $\Gamma_{2}$ on $H_{\tau}^{0}\left(\mathrm{~T}^{2}, L^{k} \otimes \delta_{\tau}\right)$.

Now consider the diagonal action of the Weyl group on $\mathrm{T}^{2}$. Its trivial lift to the half-form bundle acts holomorphically and preserves the metric. So we have a unitary representation of $W$ on $H_{\tau}^{0}\left(\mathrm{~T}^{2}, L^{k} \otimes \delta_{\tau}\right)$. It is easy to see that this 
representation commutes with the one of $\Gamma_{2}$. This gives a representation of $\Gamma_{2}$ on the subspace of alternating sections that we denote by $R_{2}^{\text {alt }}$. By Theorem 5.4, we have

Theorem 6.5. The coefficients of the matrix of $R_{2}^{\text {alt }}(S, e)$ and $R_{2}^{\text {alt }}(T, 1)$ in the basis $\chi_{\mu, k} \otimes \Omega^{1 / 2}, \mu \in \mathfrak{A} \cap k^{-1} \Lambda^{*}$, are, respectively,

$$
\frac{e(i)^{-1}}{k^{\frac{n}{2}} \operatorname{Vol}(\mathfrak{t} / \Lambda)} \sum_{w \in W}(-1)^{\ell(w)} \exp \left(-2 i \pi k B\left(\mu, w\left(\mu^{\prime}\right)\right)\right)
$$

and

$$
\delta_{\mu, \mu^{\prime}} \exp (i \pi k B(\mu, \mu))
$$

\section{Algebraic projective Representation}

Consider the category of representations of the quantum group $U_{q}(\mathfrak{g})$ with $q$ being the root of unity. This category has a subquotient, called the fusion category, which is a modular tensor category. Following [12, we can define a natural projective representation of the mapping class group of any two-dimensional surface with marked points on appropriate spaces of morphisms in this category. In particular, for the torus, we get a projective representation of the modular group that we define in this section.

7.1. The representation $R_{\infty}$. Denote by $\rho$ the half sum of positive roots and by $h^{\vee}=1+\rho\left(\alpha_{0}^{\vee}\right)$ the dual Coxeter number. Let $k$ be an integer larger than $h^{\vee}$. Then the set of admissible weights at level $k-h^{\vee}$ is

$$
C_{k}:=\Lambda^{*} \cap\left(k-h^{\vee}\right) \overline{\mathfrak{A}} .
$$

For any $\lambda, \mu \in C_{k}$, let

$$
\tilde{s}_{\lambda \mu}:=\left|\frac{\Lambda^{*}}{k \Lambda}\right|^{-1 / 2}{ }_{i}^{\left|\Delta_{+}\right|} \sum_{w \in W}(-1)^{\ell(w)} \exp \left(-\frac{2 i \pi}{k} B(w(\lambda+\rho), \mu+\rho)\right)
$$

and

$$
\tilde{t}_{\lambda \mu}:=\delta_{\lambda \mu} \exp \left(\frac{i \pi}{k} B(\lambda, \lambda+2 \rho)\right)
$$

Consider the action of the modular group on the real projective line induced by the standard action on $\mathbb{R}^{2}$. Choose a base point $L_{o} \in \mathbb{P}^{1}(\mathbb{R})$. Let $\Gamma_{\infty}$ be the set of pairs $(A, \gamma)$, where $A \in \Gamma$ and $\gamma$ is a homotopy class (with fixed endpoint) of a path in $\mathbb{P}^{1}(\mathbb{R})$ from $A . L_{o}$ to $L_{o} . \Gamma_{\infty}$ is an extension by $\mathbb{Z}$ of the modular group, the product being given by

$$
(A, \gamma) \cdot\left(A^{\prime}, \gamma^{\prime}\right)=\left(A A^{\prime}, A\left(\gamma^{\prime}\right) \star \gamma\right) .
$$

Assume that $L_{o}=[1,0]$. Then $\Gamma_{\infty}$ is generated by $\hat{s}=(S,[\phi]), \hat{t}=(T,[1,0])$ and $\hat{\gamma}=(\mathrm{id},[\gamma])$, where $\phi$ and $\gamma$ are the paths

$$
\phi(t)=\left[\sin \left(t \frac{\pi}{2}\right), \cos \left(t \frac{\pi}{2}\right)\right], \quad \gamma(t)=[\cos (t \pi), \sin (t \pi)],
$$

with $0 \leqslant t \leqslant 1$.

Theorem 7.1. $\Gamma_{\infty}$ admits a representation $R_{\infty}$ on $\mathbb{C}^{C_{k}}$ determined by

$$
R_{\infty}(\hat{s})=\left(\exp \left(-i \frac{\pi}{4} c\right) \tilde{s}_{\lambda \mu}\right), \quad R_{\infty}(\hat{t})=\left(\tilde{t}_{\lambda \mu}\right), \quad R_{\infty}(\hat{\gamma})=\left(\exp \left(i \frac{\pi}{2} c\right) \delta_{\lambda \mu}\right) .
$$


The reader is referred to the book of Bakalov-Kirillov [1 for a detailed exposition on modular tensor category and the fusion category of $U_{q}(\mathfrak{g})$. The formulas for $s_{\lambda \mu}$ and $t_{\lambda \mu}$ are given in theorem 3.3.20. That they give a projective representation is the content of chapter 3.1; cf. remark 3.1.9. The definition of the central extension is in chapter 5.7, the case of the torus being treated in example 5.7.7. Note also that there is a misprint in the formula for $s_{\lambda \mu}$; compare with proposition 3.8 of $[9]$.

7.2. Comparison of the representations $R_{2}^{\text {alt }}$ and $R_{\infty}$. To compare $R_{2}^{\text {alt }}$ with $R_{\infty}$, we introduce a third extension $\Gamma_{4}$ of the modular group. By definition, $\Gamma_{4}$ consists of the pairs $(A, e)$, where $A \in \Gamma$ and $e$ is a continuous function from $\mathcal{H}_{+}$to $\mathbb{C}$ satisfying $e(\tau)^{4}=(-c \tau+d)^{2 n}$, with $a, b, c, d$ the coefficients of $A$. The product is given by the same formula as for $\Gamma_{2}$. Let $\mathbb{Z}_{4}=\{ \pm 1, \pm i\}$. The morphism

$$
\Gamma_{2} \times \mathbb{Z}_{4} \rightarrow \Gamma_{4}, \quad(A, e, u) \rightarrow(A, e u)
$$

is onto with kernel $\{(\mathrm{id}, 1,1),(\mathrm{id},-1,-1)\}$. Thus we have a representation $R_{4}$ of $\Gamma_{4}$ given by

$$
R_{4}(A, e u)=u R_{2}^{\text {alt }}(A, e), \quad(A, e) \in \Gamma_{2}, u \in \mathbb{Z}_{4} .
$$

Recall that $\Gamma_{\infty}$ is generated by $\hat{s}, \hat{t}$ and $\hat{\gamma}$.

Lemma 7.2. There exists a morphism $\Psi$ from $\Gamma_{\infty}$ onto $\Gamma_{4}$ such that

$$
\begin{array}{rlrl}
\Psi(\hat{s}) & =(S, e) \quad \text { with } & e(i)=e^{-i \frac{\pi}{4} n}, \\
\Psi(\hat{\gamma})=\left(\mathrm{id}, i^{\operatorname{dim} G}\right), & \Psi(\hat{t})=(T, 1) .
\end{array}
$$

Proof. Consider the groups $\Gamma_{\infty}^{\mathbb{R}}$ and $\Gamma_{4}^{\mathbb{R}}$ defined exactly as $\Gamma_{\infty}$ and $\Gamma_{4}$, except that we replace the modular group by $\Gamma^{\mathbb{R}}:=\mathrm{Sl}(2, \mathbb{R})$. We will construct a morphism from $\Gamma_{\infty}^{\mathbb{R}}$ onto $\Gamma_{4}^{\mathbb{R}}$ whose restriction to $\Gamma_{\infty}$ is $\Psi$. Let $j$ be the morphism from the unit circle $\mathrm{U} \subset \mathbb{C}$ into $\Gamma^{\mathbb{R}}$

$$
j(u)=\left(\begin{array}{cc}
\operatorname{Re} u & -\operatorname{Im} u \\
\operatorname{Im} u & \operatorname{Re} u
\end{array}\right) .
$$

Since $\Gamma^{\mathbb{R}}$ admits a deformation retract onto $j(\mathrm{U})$, one has a bijective correspondence between the morphisms from $\Gamma_{\infty}^{\mathbb{R}}$ to $\Gamma_{4}^{\mathbb{R}}$ covering the identity of $\Gamma^{\mathbb{R}}$ and the morphisms from $j^{*} \Gamma_{\infty}^{\mathbb{R}}$ to $j^{*} \Gamma_{4}^{\mathbb{R}}$ covering the identity of $\mathrm{U}$. On one hand

$$
j^{*} \Gamma_{4}^{\mathbb{R}} \simeq\left\{(u, e) \in \mathrm{U} \times \mathbb{C}^{*} / e^{4}=u^{-2 n}\right\}=: \mathrm{U}^{4}
$$

because for $\tau=i,-c \tau+d=u^{-1}$ if $a, b, c$, and $d$ are the coefficients of $j(u)$. On the other hand

$$
j^{*} \Gamma_{\infty}^{\mathbb{R}} \simeq\left\{(u, \theta) / \exp (i \theta)=u^{2}\right\}=: \mathrm{U}^{\infty},
$$

where we send $(u, \theta)$ into $(j(u),[\gamma])$ with $\gamma$ the path

$$
\gamma(t)=\left[\cos \left(\frac{1-t}{2} \theta\right), \sin \left(\frac{1-t}{2} \theta\right)\right], \quad t \in[0,1] .
$$

In particular, $\hat{s}$ and $\hat{\gamma}$ are identified with $(i, \pi)$ and $(1,-2 \pi)$. The morphism we are looking for is

$$
\mathrm{U}^{\infty} \rightarrow \mathrm{U}^{4}, \quad(u, \theta) \rightarrow\left(u, u^{\left|\Delta_{+}\right|} e^{-i \frac{\theta}{4} \operatorname{dim} G}\right) .
$$

Here $\left|\Delta_{+}\right|$is the number of positive roots of $G$. The images of $\hat{s}$ and $\hat{\gamma}$ are given by a straightforward computation using that $\operatorname{dim} G=2\left|\Delta_{+}\right|+n$. 
Lemma 7.3. For any $k$, there is a morphism $\zeta_{k}: \Gamma_{\infty} \rightarrow \mathbb{C}^{*}$ such that

$$
\zeta_{k}(\hat{s})=e^{i \frac{\pi}{4} x_{k}}, \quad \zeta_{k}(\hat{t})=e^{-i \frac{\pi}{12} x_{k}}, \quad \zeta_{k}(\hat{\gamma})=e^{-i \frac{\pi}{2} x_{k}},
$$

with $x_{k}=h^{\vee} \operatorname{dim} G / k$, where $h^{\vee}$ is the dual Coxeter number of $G$.

Proof. The value of $x_{k}$ does not matter for the proof. We only use that $\Gamma_{\infty}$ is generated by $\hat{s}, \hat{t}$ and $\hat{\gamma}$ with the relations

$$
\left(\hat{\gamma} \hat{s}^{2}\right)^{2}=\mathrm{id}, \quad(\hat{s} \hat{t})^{3}=\hat{s}^{2}, \quad \hat{\gamma} \text { and } \hat{s}^{2} \text { are central, }
$$

as it is asserted in [1, example 5.7.7.

So by the previous lemmas, we define a representation of $\Gamma_{\infty}$ by

$$
\tilde{R}_{\infty}(A, \gamma)=\zeta_{k}(A, \gamma) \cdot R_{4}(\Psi(A, \gamma)) .
$$

We will prove it is the same as $R_{\infty}$. To do this we have to identify $\mathbb{C}^{C_{k}}$ with $H_{\tau}^{0}\left(\mathrm{~T}^{2}, L^{k} \otimes \delta_{\tau}\right)$. We need the following well-known fact.

Lemma 7.4. For any $k$, the map sending $\lambda$ into $\frac{\lambda+\rho}{k}$ is a bijection between $C_{k}$ and $\mathfrak{A} \cap k^{-1} \Lambda^{*}$

The identification is then defined by sending the canonical basis of $\mathbb{C}^{C_{k}}$ into the basis $\chi_{\mu, k} \otimes \Omega^{1 / 2}, \mu \in \mathfrak{A} \cap k^{-1} \Lambda^{*}$.

Theorem 7.5. The matrices of $\tilde{R}_{\infty}(\hat{s}), \tilde{R}_{\infty}(\hat{t}), \tilde{R}_{\infty}(\hat{\gamma})$ in the basis $\chi_{(\lambda+\rho) / k, k} \otimes$ $\Omega^{1 / 2}, \lambda \in C_{k}$, are respectively given by

$$
\left(\exp \left(-i \frac{\pi}{4} c\right) \tilde{s}_{\lambda \mu}\right), \quad\left(\tilde{t}_{\lambda \mu}\right), \quad\left(\exp \left(i \frac{\pi}{2} c\right) \delta_{\lambda \mu}\right),
$$

where $\left(s_{\lambda \mu}\right)$ and $\left(t_{\lambda \mu}\right)$ are the matrices defined in section 7 .

Not only does it prove that $\tilde{R}_{\infty}=R_{\infty}$, but it also proves Theorem 7.1

Proof. This follows from Theorem 6.5. Since $\Psi(\hat{s})=(S, e)$ with $e(i)=e^{-i \frac{\pi}{4}}$ and $\zeta_{k}(\hat{s})=\exp \left(i \frac{\pi}{4} \operatorname{dim} G \frac{h^{\vee}}{k}\right)$, the matrix of $\tilde{R}_{\infty}(\hat{s})$ is

$$
\begin{aligned}
& \frac{e^{i \frac{\pi}{4}\left(n+\frac{h^{\vee}}{k} \operatorname{dim} G\right)}}{k^{\frac{n}{2}} \operatorname{Vol}(\mathfrak{t} / \Lambda)} \sum_{w \in W}(-1)^{\ell(w)} \exp \left(-2 i \pi k B\left(\frac{\rho+\mu}{k}, w\left(\frac{\rho+\lambda}{k}\right)\right)\right. \\
= & e^{i \frac{\pi}{4}\left(n+\frac{h^{\vee}}{k} \operatorname{dim} G\right)}\left|\frac{\Lambda^{*}}{k \Lambda}\right|^{-1 / 2} \sum_{w \in W}(-1)^{\ell(w)} \exp \left(-2 i \pi k^{-1} B(\rho+\mu, w(\rho+\lambda))\right)
\end{aligned}
$$

because

$$
\left|\frac{\Lambda^{*}}{\Lambda}\right|=\frac{\operatorname{Vol}(\mathfrak{t} / \Lambda)}{\operatorname{Vol}\left(\mathfrak{t} / \Lambda^{*}\right)}=\operatorname{Vol}^{2}(\mathfrak{t} / \Lambda)
$$

To conclude, it suffices to compare with the formula defining $\tilde{s}_{\lambda \mu}$. Since $\Psi(\hat{t})=$ $(T, 1)$ and because of the value of $\zeta_{k}(\hat{t})$, the matrix of $\tilde{R}_{\infty}(\hat{t})$ is

$$
\begin{aligned}
& e^{-i \frac{\pi}{12} \operatorname{dim} G \frac{h^{\vee}}{k}} \delta_{\mu, \lambda} \exp \left(i \pi k B\left(\frac{\rho+\mu}{k}, \frac{\rho+\mu}{k}\right)\right) \\
= & \delta_{\mu, \lambda} \exp \left(i \pi k^{-1} B(\mu, 2 \rho+\mu)\right),
\end{aligned}
$$

where we have used that

$$
\frac{B(\rho, \rho)}{2 k}=\frac{1}{24}(\operatorname{dim} G-c),
$$

which follows from Freudenthal's strange formula. 


\section{Complex StruCture DePENDENCE IN THE SEMI-ClASSICAL Limit}

8.1. Fourier integral operators. Let $M$ be a symplectic compact manifold with a prequantization bundle $L$. Consider two pairs $\left(j_{a}, \delta_{a}\right)$ and $\left(j_{b}, \delta_{b}\right)$ consisting of a positive complex structure of $M$ together with a half-form bundle. We say that a section $\varphi$ of $\operatorname{Hom}\left(\delta_{a}, \delta_{b}\right)$ is a half-form bundle isomorphism if its square at $x$ is given by

$$
\varphi_{x}^{\otimes 2}=\pi_{b, a, x}^{*}: \bigwedge^{\text {top }} E_{a, x}^{*} \rightarrow \bigwedge_{\text {top }}^{*} E_{b, x}^{*}
$$

where $\pi_{b, a, x}$ is the projection from $E_{b, x}:=T_{x}^{1,0}\left(M, j_{b}\right)$ to $E_{a, x}:=T_{x}^{1,0}\left(M, j_{a}\right)$ with kernel $\bar{E}_{b, x}$.

For $c=a, b$, denote by $\mathcal{H}_{k}(c)$ the space of sections of $L^{k} \otimes \delta_{c}$ holomorphic with respect to $j_{c}$. Consider a sequence $\left(S_{k}\right)$ such that for every $k, S_{k}$ is an operator $\mathcal{H}_{k}(a) \rightarrow \mathcal{H}_{k}(b)$. The scalar product of $\mathcal{H}_{k}(a)$ gives us an isomorphism

$$
\operatorname{Hom}\left(\mathcal{H}_{k}(a), \mathcal{H}_{k}(b)\right) \simeq \mathcal{H}_{k}(b) \otimes \overline{\mathcal{H}}_{k}(a) .
$$

The latter space can be regarded as the space of holomorphic sections of

$$
\left(L^{k} \otimes \delta_{b}\right) \otimes\left(\bar{L}^{k} \otimes \bar{\delta}_{a}\right) \rightarrow M^{2},
$$

where $M^{2}$ is endowed with the complex structure $\left(j_{b},-j_{a}\right)$. The section $S_{k}(x, y)$ associated in this way to $S_{k}$ is its Schwartz kernel.

We say that $\left(S_{k}\right)$ is a Fourier integral operator with its symbol the half-form bundle isomorphism $\varphi$ if

$$
S_{k}(x, y)=\left(\frac{k}{2 \pi}\right)^{n} E^{k}(x, y) f(x, y, k)+O\left(k^{-\infty}\right),
$$

where

- $E$ is a section of $L \otimes \bar{L} \rightarrow M^{2}$ such that $\|E(x, y)\|<1$ if $x \neq y$,

$$
E(x, x)=u \otimes \bar{u}, \quad \forall u \in L_{x} \text { such that }\|u\|=1,
$$

and $\bar{\partial} E \equiv 0$ modulo a section vanishing to any order along the diagonal.

- $f(., k)$ is a sequence of sections of $\delta_{b} \otimes \bar{\delta}_{a} \rightarrow M^{2}$ which admits an asymptotic expansion in the $\mathcal{C}^{\infty}$ topology of the form

$$
f(., k)=f_{0}+k^{-1} f_{1}+k^{-2} f_{2}+\ldots
$$

whose coefficients satisfy $\bar{\partial} f_{i} \equiv 0$ modulo a section vanishing to any order along the diagonal.

- The restriction to the diagonal of the leading coefficient $f_{0}$ is equal to $\varphi$ if we identify $\delta_{b} \otimes \bar{\delta}_{a}$ with $\operatorname{Hom}\left(\delta_{a}, \delta_{b}\right)$ using the metric of $\delta_{a}$.

8.2. The maps $\Psi_{\tau_{1}, \tau_{2}, k}$ in the semi-classical limit. Recall that for any $\tau \in \mathcal{H}_{+}$, we defined a complex structure on $\mathrm{T}^{2}$ together with a half-form bundle $\delta_{\tau}$. Consider the morphism $\varphi_{\tau_{1}, \tau_{2}}$ from $\delta_{\tau_{1}}$ to $\delta_{\tau_{2}}$ given by

$$
\varphi_{\tau_{1}, \tau_{2}}\left(\Omega^{1 / 2}\right)=\left(\frac{\tau_{1}-\bar{\tau}_{1}}{\tau_{2}-\bar{\tau}_{1}}\right)^{n / 2} \Omega^{1 / 2}
$$

where the square root is determined in such a way that it depends continuously on $\tau_{1}, \tau_{2}$ and equal to 1 when $\tau_{1}=\tau_{2}$.

Lemma 8.1. The map $\varphi_{\tau_{1}, \tau_{2}}$ is a half-form bundle isomorphism. 
Proof. Introduce an orthonormal basis $\left(u_{i}\right)$ of $\mathfrak{t}$ and denote by $\left(p_{i}\right)$ and $\left(q_{i}\right)$ the associated linear coordinates of $\mathfrak{t}^{2}$. Let $\zeta_{\tau}^{i}$ be the complex coordinate $p_{i}+\tau q_{i}$. Let $E_{\tau}=\operatorname{span}\left(\partial_{\zeta_{\tau}^{1}}, \ldots, \partial_{\zeta_{\tau}^{n}}\right)$ be the space of vector of type $(1,0)$ with respect to the complex structure $j_{\tau}$. Let $\pi_{\tau_{2}, \tau_{1}}$ be the projection from $E_{\tau_{2}}$ to $E_{\tau_{1}}$ with kernel $\bar{E}_{\tau_{2}}$. One has

which implies that

$$
d \zeta_{\tau_{2}}^{i}=\left(\frac{\tau_{2}-\bar{\tau}_{1}}{\tau_{1}-\bar{\tau}_{1}}\right) d \zeta_{\tau_{1}}^{i}+\left(\frac{\tau_{1}-\tau_{2}}{\tau_{1}-\bar{\tau}_{1}}\right) d \bar{\zeta}_{\tau_{1}}^{i}
$$

and then that

$$
\pi_{\tau_{2}, \tau_{1}}^{*} d \zeta_{\tau_{1}}^{i}=\left(\frac{\tau_{1}-\bar{\tau}_{1}}{\tau_{2}-\bar{\tau}_{1}}\right) d \zeta_{\tau_{2}}^{i}
$$

$$
\pi_{\tau_{2}, \tau_{1}}^{*}\left(d \zeta_{\tau_{1}}^{1} \wedge \ldots \wedge d \zeta_{\tau_{1}}^{n}\right)=\left(\frac{\tau_{1}-\bar{\tau}_{1}}{\tau_{2}-\bar{\tau}_{1}}\right)^{n} d \zeta_{\tau_{2}}^{1} \wedge \ldots \wedge d \zeta_{\tau_{2}}^{n} .
$$

Finally, recall that by equation (25) the squaring map of $\delta_{\tau}$ sends $\Omega^{1 / 2}$ into $\pm \operatorname{Vol}(\mathfrak{t} / \Lambda) d \zeta_{\tau}^{1} \wedge \ldots \wedge d \zeta_{\tau}^{n}$

Theorem 8.2. For any $\tau_{1}, \tau_{2} \in \mathcal{H}_{+}$, the sequence $\left(\Psi_{\tau_{1}, \tau_{2}, k}\right)_{k}$ is a Fourier integral operator with symbol $\varphi_{\tau_{1}, \tau_{2}}$.

Section 8.3 is devoted to the proof.

8.3. Proof of Theorem 8.2. As previously, we lift everything from $T^{2}$ to $\mathfrak{t}^{2}$. Denote by $p_{2}, q_{2}$ (resp. $p_{1}, q_{1}$ ) the coordinates on the left (resp. right) factor of $\mathfrak{t}^{2} \times \mathfrak{t}^{2}$. Let $\zeta_{i}=p_{i}+\tau_{i} q_{i}$ for $i=1,2$.

Let us first compute the section $E$ and the leading coefficient $f_{0}$ of (41). Let $L_{\mathfrak{t}^{2}}$ be the trivial line bundle over $\mathfrak{t}^{2}$ with the connection defined in section 3 . Consider the bundle $L_{\mathfrak{t}^{2}} \otimes \bar{L}_{\mathfrak{t}^{2}}$ endowed with the holomorphic structure compatible with the connection and the complex structure $\left(j_{\tau_{2}},-j_{\tau_{1}}\right)$ of $\mathfrak{t}^{2} \times \mathfrak{t}^{2}$.

Lemma 8.3. The section of $L_{\mathfrak{t}^{2}} \otimes \bar{L}_{\mathfrak{t}^{2}}$,

$$
\exp \left(\frac{-i \pi}{\tau_{2}-\bar{\tau}_{1}} B\left(\zeta_{2}-\bar{\zeta}_{1}, \zeta_{2}-\bar{\zeta}_{1}\right)\right) s_{\tau_{2}} \otimes \bar{s}_{\tau_{1}}
$$

is holomorphic and restricts to the constant section equal to 1 on the diagonal. The morphism $\varphi_{\tau_{1}, \tau_{2}}$ is sent to the constant section equal to

$$
\left(\frac{2 i \pi}{\tau_{2}-\overline{\tau_{1}}}\right)^{n / 2} \operatorname{Vol}(\mathfrak{t} / \Lambda) \Omega^{1 / 2} \otimes \bar{\Omega}^{1 / 2}
$$

by the isomorphism between $\operatorname{Hom}\left(\delta_{\tau_{1}}, \delta_{\tau_{2}}\right)$ and $\delta_{\tau_{2}} \otimes \bar{\delta}_{\tau_{1}}$ induced by the metric of $\delta_{\tau_{1}}$.

The first part is proved by a straightforward computation using (4) and the second part follows from Lemma 6.1. By Theorem [6.2, the Schwartz kernel of $\Psi_{\tau_{1}, \tau_{2}, k}$ lifts from $\mathrm{T}^{2} \times \mathrm{T}^{2}$ to $\mathfrak{t}^{2} \times \mathfrak{t}^{2}$ into

$$
\begin{aligned}
S_{k}\left(p_{2}, q_{2}, p_{1}, q_{1}\right) & =\sum_{\mu \in\left(k^{-1} \Lambda^{*}\right) / \Lambda} \Theta_{\mu, k}^{\tau_{2}}\left(p_{2}, q_{2}\right) \bar{\Theta}_{\mu, k}^{\tau_{1}}\left(p_{1}, q_{1}\right)\left(s_{\tau_{2}}^{k} \Omega^{1 / 2}\right) \otimes\left(\bar{s}_{\tau_{1}}^{k} \bar{\Omega}^{1 / 2}\right) \\
& =\sum_{\substack{\mu \in\left(k^{-1} \Lambda^{*}\right) / \Lambda \\
\gamma_{1}, \gamma_{2} \in \mu+\Lambda}} f_{\gamma_{2}, \gamma_{1}}\left(p_{2}, q_{2}, p_{1}, q_{1}\right)\left(s_{\tau_{2}}^{k} \Omega^{1 / 2}\right) \otimes\left(\bar{s}_{\tau_{1}}^{k} \bar{\Omega}^{1 / 2}\right),
\end{aligned}
$$


where the coefficients are given by

$$
f_{\gamma_{2}, \gamma_{1}}=\exp \left(2 i \pi k\left(\frac{\tau_{2}}{2} B\left(\gamma_{2}, \gamma_{2}\right)-B\left(\zeta_{2}, \gamma_{2}\right)-\frac{\bar{\tau}_{1}}{2} B\left(\gamma_{1}, \gamma_{1}\right)+B\left(\bar{\zeta}_{1}, \gamma_{1}\right)\right)\right) .
$$

Lemma 8.4. For any compact set $K$ of $\mathfrak{t}$ such that $K \cap \Lambda=\emptyset$, there exists $C>0$ such that for all $p_{2}, q_{2}, p_{1}, q_{1}$ satisfying $q_{1}-q_{2} \notin K$ one has

$$
\left|S_{k}\left(p_{2}, q_{2}, p_{1}, q_{1}\right)\right| \leqslant C e^{-k / C} .
$$

This shows that the sequence of Schwartz kernels of $\Psi_{\tau_{1}, \tau_{2}, k}$ is a $O\left(k^{-\infty}\right)$ outside the diagonal. For the proof of the lemma we need the following general estimates.

Lemma 8.5. For any $C>0$ there exists $C^{\prime}>0$ such that

$$
\sum_{\gamma \in \mathbb{Z}^{n}} e^{-k C|\gamma-x|^{2}} \leqslant C^{\prime} k^{n / 2}, \quad \forall x \in \mathbb{R}^{n} .
$$

For any $C>0$, for any compact $K$ of $\mathbb{R}^{n}$ and for any subset $P$ of $\mathbb{Z}^{n}$ such that $K \cap\left(\mathbb{Z}^{n} \backslash P\right)=\emptyset$, there exists $C^{\prime}>0$ such that

$$
\sum_{\gamma \in \mathbb{Z}^{n} \backslash P} e^{-k C|\gamma-x|^{2}} \leqslant C^{\prime} e^{-k / C^{\prime}}, \quad \forall x \in K
$$

Proof of Lemma 8.4, By a straightforward computation we deduce from (18) and (44) that

$$
\left|f_{\gamma_{2}, \gamma_{1}} s_{\tau_{2}}^{k} \otimes \bar{s}_{\tau_{1}}^{k}\right|=\exp \left(-k \pi\left(\frac{\tau_{2}-\bar{\tau}_{2}}{2 i}\left|q_{2}-\gamma_{2}\right|^{2}+\frac{\tau_{1}-\bar{\tau}_{1}}{2 i}\left|q_{1}-\gamma_{1}\right|^{2}\right)\right) .
$$

Hence for some positive $C$,

$$
\left|f_{\gamma_{2}, \gamma_{1}} s_{\tau_{2}}^{k} \otimes \bar{s}_{\tau_{1}}^{k}\right| \leqslant e^{-k C\left(\left|\gamma_{2}-q_{2}\right|^{2}+\left|\left(q_{2}-q_{1}\right)-\left(\gamma_{2}-\gamma_{1}\right)\right|^{2}\right)} .
$$

So with $q=q_{2}-q_{1}$,

$$
\begin{aligned}
\sum_{\gamma_{1}, \gamma_{2} \in \mu+\Lambda}\left|f_{\gamma_{2}, \gamma_{1}} s_{\tau_{2}}^{k} \otimes \bar{s}_{\tau_{1}}^{k}\right| & \leqslant \sum_{\substack{\gamma_{2} \in \mu+\Lambda \\
\gamma \in \Lambda}} e^{-k C\left(\left|\gamma_{2}-q_{2}\right|^{2}+|q-\gamma|^{2}\right)} \\
& \leqslant\left(\sum_{\gamma \in \Lambda} e^{-k C\left|\mu+\gamma-q_{2}\right|^{2}}\right)\left(\sum_{\gamma \in \Lambda} e^{-k C|\gamma-q|^{2}}\right) .
\end{aligned}
$$

By Lemma 8.5, there exists $C^{\prime}>0$ such that the first factor is bounded by $C^{\prime} k^{n / 2}$, the second one by $C^{\prime} e^{-k / C^{\prime}}$, and these estimates are uniform with respect to $\mu, q_{1}$ and $q_{2}$ such that $q_{1}-q_{2} \in K$. Since the cardinal of $\left(k^{-1} \Lambda^{*}\right) / \Lambda$ is $k^{n}\left|\Lambda^{*} / \Lambda\right|$, we obtain with a larger $C^{\prime}$ that

$$
\sum_{\substack{\mu \in\left(k^{-1} \Lambda^{*}\right) / \Lambda \\ \gamma_{1}, \gamma_{2} \in \mu+\Lambda}}\left|f_{\gamma_{2}, \gamma_{1}}\left(p_{2}, q_{2}, p_{1}, q_{1}\right) s_{\tau_{2}}^{k} \otimes \bar{s}_{\tau_{1}}^{k}\right| \leqslant C^{\prime} e^{-k / C^{\prime}},
$$

which proves the result.

Using exactly the same method and Lemma 8.5 with $P=\{0\}$, we show

Lemma 8.6. There exists $C>0$ such that

$$
\sum_{\substack{\mu \in\left(k^{-1} \Lambda^{*}\right) / \Lambda \\ \gamma_{1}, \gamma_{2} \in \mu+\Lambda, \gamma_{1} \neq \gamma_{2}}}\left|f_{\gamma_{2}, \gamma_{1}}\left(p_{2}, q_{2}, p_{1}, q_{1}\right) s_{\tau_{2}}^{k} \otimes \bar{s}_{\tau_{1}}^{k}\right| \leqslant C e^{-k / C}
$$

for all $p_{2}, q_{2}, p_{1}$ and $q_{1}$ such that $\left|q_{1}-q_{2}\right| \leqslant R / 2$ with $R=\min \{|\gamma|, \gamma \in \Lambda \backslash\{0\}\}$. 
So up to an $O\left(k^{-\infty}\right), S_{k}\left(p_{2}, q_{2}, p_{1}, q_{1}\right)$ is given on a neighborhood of the diagonal by the sum of the $f_{\gamma, \gamma}$ where $\gamma$ runs over $k^{-1} \Lambda^{*}$.

Lemma 8.7. We have

$$
\begin{aligned}
\sum_{\gamma \in k^{-1} \Lambda^{*}} f_{\gamma, \gamma}\left(p_{2}, q_{2}, p_{1}, q_{1}\right)= & \left(\frac{i k}{\tau_{2}-\bar{\tau}_{1}}\right)^{n / 2} \operatorname{Vol}(\mathfrak{t} / \Lambda) \\
& \times \sum_{\lambda \in \Lambda} \exp \left(\frac{-i \pi k}{\tau_{2}-\bar{\tau}_{1}} B\left(\lambda+\zeta_{2}-\bar{\zeta}_{1}, \lambda+\zeta_{2}-\bar{\zeta}_{1}\right)\right) .
\end{aligned}
$$

Proof. Introduce a basis $\left(\pi_{i}\right)$ of $\Lambda^{*}$. Let $\gamma \in k^{-1} \Lambda^{*}$ and write $k \gamma=\sum x_{i} \pi_{i}$. One has

$$
\begin{aligned}
f_{\gamma, \gamma}\left(p_{2}, q_{2}, p_{1}, q_{1}\right) & =\exp \left(-\left(\frac{\tau_{2}-\bar{\tau}_{1}}{2 i}\right)\left(\frac{2 \pi}{k}\right) B(k \gamma, k \gamma)-2 i \pi B\left(\zeta_{2}-\bar{\zeta}_{1}, k \gamma\right)\right) \\
& =u(x) \exp (-i\langle\eta, x\rangle),
\end{aligned}
$$

where $u$ is the complex-valued function of $\mathbb{R}^{n}$ given by

$$
u(x)=\exp \left(-\frac{1}{2}\left(\frac{\tau_{2}-\bar{\tau}_{1}}{i}\right)\left(\frac{2 \pi}{k}\right) \sum_{i, j} B\left(\pi_{i}, \pi_{j}\right) x_{i} x_{j}\right)
$$

and

$$
\eta_{i}=2 \pi B\left(\zeta_{2}-\bar{\zeta}_{1}, \pi_{i}\right)
$$

Let $\left(\gamma_{i}\right)$ be the dual basis of $\left(\pi_{i}\right)$. Then using that $B\left(\gamma_{i}, \gamma_{j}\right)$ is the inverse of $B\left(\pi_{i}, \pi_{j}\right)$ and that the determinant of $\left(B\left(\gamma_{i}, \gamma_{j}\right)\right)_{i, j}$ is the square of the volume of $\mathfrak{t} / \Lambda$, we prove that the Fourier transform of $u$ is

$$
\hat{u}(\xi)=\left(\frac{i k}{\tau_{2}-\bar{\tau}_{1}}\right)^{n / 2} \operatorname{Vol}(\mathfrak{t} / \Lambda) \exp \left(-\frac{1}{2}\left(\frac{i}{\tau_{2}-\bar{\tau}_{1}}\right)\left(\frac{k}{2 \pi}\right) \sum_{i, j} B\left(\gamma_{i}, \gamma_{j}\right) \xi_{i} \xi_{j}\right) .
$$

The Fourier transform of $v(x)=u(x) \exp (-i\langle\eta, x\rangle)$ is

$$
\hat{v}(\xi)=\hat{u}(\xi+\eta)
$$

for real $\eta$. This is also verified for any $\eta \in \mathbb{C}^{n}$ by analytic prolongation. By Poisson's summation formula,

$$
\sum_{x \in \mathbb{Z}^{n}} v(x)=\sum_{\xi \in \mathbb{Z}^{n}} \hat{v}(2 \pi \xi)
$$

Let us compute $\hat{v}(2 \pi \xi)$. Since $\sum \eta_{i} \gamma_{i}=2 \pi\left(\zeta_{2}-\bar{\zeta}_{1}\right)$, we have

$$
\sum_{i, j} B\left(\gamma_{i}, \gamma_{j}\right)\left(2 \pi \xi_{i}+\eta_{i}\right)\left(2 \pi \xi_{j}+\eta_{j}\right)=(2 \pi)^{2} B\left(\lambda+\zeta_{2}-\bar{\zeta}_{1}, \lambda+\zeta_{2}-\bar{\zeta}_{1}\right),
$$

where $\lambda=\sum \xi_{i} \gamma_{i} \in \Lambda$. So $\hat{v}(2 \pi \xi)$, which is equal to $\hat{u}(2 \pi \xi+\eta)$, is given by

$$
\hat{v}(2 \pi \xi)=\left(\frac{i k}{\tau_{2}-\bar{\tau}_{1}}\right)^{n / 2} \operatorname{Vol}(\mathfrak{t} / \Lambda) \exp \left(\frac{-i \pi k}{\tau_{2}-\bar{\tau}_{1}} B\left(\lambda+\zeta_{2}-\bar{\zeta}_{1}, \lambda+\zeta_{2}-\bar{\zeta}_{1}\right)\right),
$$

which concludes the proof.

Lemma 8.8. There exists $\epsilon>0$ and $C>0$ such that

$$
\sum_{\lambda \in \Lambda \backslash\{0\}}\left|\exp \left(\frac{-i \pi k}{\tau_{2}-\bar{\tau}_{1}} B\left(\lambda+\zeta_{2}-\bar{\zeta}_{1}, \lambda+\zeta_{2}-\bar{\zeta}_{1}\right)\right) s_{\tau_{2}}^{k} \otimes \bar{s}_{\tau_{1}}^{k}\right| \leqslant C e^{-k / C}
$$

for all $p_{2}, q_{2}, p_{1}$ and $q_{1}$ satisfying $\left|q_{1}-q_{2}\right| \leqslant \epsilon$ and $\left|p_{1}-p_{2}\right| \leqslant \epsilon$. 
Proof. With a straightforward computation using (18), we obtain that

$$
\left|\exp \left(\frac{-i \pi}{\tau_{2}-\bar{\tau}_{1}}\left|\lambda+\zeta_{2}-\bar{\zeta}_{1}\right|^{2}\right) s_{\tau_{2}} \otimes \bar{s}_{\tau_{1}}\right|^{2}=e^{-\left(a|\mu|^{2}+2 b B(\mu, q)+c|q|^{2}\right)},
$$

where $q=q_{2}-q_{1}, \mu=\lambda+p_{2}-p_{1}$ and $a, b$ and $c$ are the real numbers

$$
\begin{aligned}
a & =\frac{-i \pi}{\left|\tau_{2}-\bar{\tau}_{1}\right|^{2}}\left(\tau_{2}-\bar{\tau}_{2}+\tau_{1}-\bar{\tau}_{1}\right), \\
b & =\frac{i \pi}{\left|\tau_{2}-\bar{\tau}_{1}\right|^{2}}\left(\bar{\tau}_{2} \bar{\tau}_{1}-\tau_{2} \tau_{1}\right), \\
c & =\frac{-i \pi}{\left|\tau_{2}-\bar{\tau}_{1}\right|^{2}}\left(\left|\tau_{2}\right|^{2}\left(\tau_{1}-\bar{\tau}_{1}\right)+\left|\tau_{1}\right|^{2}\left(\tau_{2}-\bar{\tau}_{2}\right)\right) .
\end{aligned}
$$

Write

$$
a|\mu|^{2}+2 b B(\mu, q)+c|q|^{2}=a\left|\mu+\frac{b}{a} q\right|^{2}+\left(c-\frac{b^{2}}{a}\right)|q|^{2} .
$$

Using that $a$ is positive, one proves that there exists $\epsilon>0$ such that

$$
|q| \leqslant \epsilon \text { and }\left|p_{2}-p_{1}\right| \leqslant \epsilon \Rightarrow \frac{a}{2}\left|\mu+\frac{b}{a} q\right|^{2}+\left(c-\frac{b^{2}}{a}\right)|q|^{2} \geqslant 0
$$

for any nonvanishing $\lambda \in \Lambda$. So

$$
\sum_{\lambda \in \Lambda \backslash\{0\}}\left|\exp \left(\frac{-i k \pi}{\tau_{2}-\bar{\tau}_{1}}\left|\lambda+\zeta_{2}-\bar{\zeta}_{1}\right|^{2}\right) s_{\tau_{2}}^{k} \otimes \bar{s}_{\tau_{1}}^{k}\right| \leqslant \sum_{\lambda \in \Lambda \backslash\{0\}} e^{-\frac{a}{4} k\left|\lambda+p_{2}-p_{1}+\frac{b}{a} q\right|^{2}}
$$

when $|q|$ and $\left|p_{2}-p_{1}\right|$ are smaller than $\epsilon$. We conclude with Lemma 8.5.

Collecting together the previous lemmas, we obtain

$$
\begin{aligned}
& S_{k}\left(p_{2}, q_{2}, p_{1}, q_{1}\right)=\left(\frac{k}{2 \pi}\right)^{n}\left(\frac{2 i \pi}{\tau_{2}-\bar{\tau}_{1}}\right)^{n / 2} \operatorname{Vol}(\mathfrak{t} / \Lambda) \\
& \quad \times \exp \left(-\frac{i \pi k}{\tau_{2}-\bar{\tau}_{1}} B\left(\zeta_{2}-\bar{\zeta}_{1}, \zeta_{2}-\bar{\zeta}_{1}\right)\right)\left(s_{\tau_{2}}^{k} \Omega^{1 / 2}\right) \otimes\left(\bar{s}_{\tau_{1}}^{k} \bar{\Omega}^{1 / 2}\right)+R_{k}\left(p_{2}, q_{2}, p_{1}, q_{1}\right),
\end{aligned}
$$

where the remainder satisfies for some $\epsilon>0$ and $C>0$,

$$
\left|q_{1}-q_{2}\right|,\left|p_{1}-p_{2}\right| \leqslant \epsilon \Rightarrow\left|R_{k}\left(p_{2}, q_{2}, p_{1}, q_{1}\right)\right| \leqslant C e^{-k / C} .
$$

Using Lemma 8.3 , this proves Theorem 8.2 ,

\section{Asymptotic Properties of the QUANTUM REPRESENTATIONS}

9.1. Definitions. Let $M$ be a symplectic compact manifold with a positive complex structure $j$, a prequantization bundle $L$ and a half-form bundle $\delta$. Consider a symplectomorphism $\Phi: M \rightarrow M$ together with automorphisms $\Phi_{L}$ and $\varphi$ of the bundles $L$ and $\delta$, respectively, which lift $\Phi$. We assume that $\Phi_{L}$ preserves the connection and metric of $L$.

Let $\mathcal{H}_{k}$ be the space of holomorphic sections of $L^{k} \otimes \delta$. Consider a family $\left(S_{k}\right)$ such that for every $k, S_{k}$ is an operator $\mathcal{H}_{k} \rightarrow \mathcal{H}_{k}$. The Schwartz kernel of $S_{k}$ is a holomorphic section of

$$
\left(L^{k} \otimes \delta\right) \otimes\left(\bar{L}^{k} \otimes \bar{\delta}\right) \rightarrow M^{2}
$$


where $M^{2}$ is endowed with the complex structure $(j,-j)$. We say that $\left(S_{k}\right)$ is a Fourier integral operator associated to $\Phi_{L}$ with symbol $\varphi$ if the Schwartz kernel sequence is of the form

$$
S_{k}(x, y)=\left(\frac{k}{2 \pi}\right)^{n} F^{k}(x, y) g(x, y, k)+O\left(k^{-\infty}\right),
$$

where:

- $F$ is a section of $L \otimes \bar{L} \rightarrow M^{2}$ such that $\|F(x, y)\|<1$ if $x \neq \Phi(y)$,

$$
F(\Phi(x), x)=\Phi_{L}(u) \otimes \bar{u}, \quad \forall u \in L_{x} \text { such that }\|u\|=1,
$$

and $\bar{\partial} F \equiv 0$ modulo a section vanishing to any order along the graph of $\Phi^{-1}$.

- $g(., k)$ is a sequence of sections of $\delta \otimes \bar{\delta} \rightarrow M^{2}$ which admits an asymptotic expansion in the $\mathcal{C}^{\infty}$ topology of the form

$$
g(., k)=g_{0}+k^{-1} g_{1}+k^{-2} g_{2}+\ldots
$$

whose coefficients satisfy $\bar{\partial} g_{i} \equiv 0$ modulo a section vanishing to any order along the graph of $\Phi^{-1}$.

- The restriction to the diagonal of the leading coefficient $g_{0}$ is equal to $\varphi$ if we identify $\delta \otimes \bar{\delta}$ with $\operatorname{Hom}(\delta, \delta)$ using the metric of $\delta$.

Let us explain the relation with the Fourier integral operators of section 8.1 . Let $\Phi(j)$ be the complex structure obtained by pushing forward $j$ with $\Phi$. Consider a half-form bundle $\delta^{\prime}$ of the complex manifold $(M, \Phi(j))$ together with an isomorphism $\varphi_{1}: \delta \rightarrow \delta^{\prime}$ whose square is equal to

$$
\varphi_{1, x}^{\otimes 2}=\left(\left(T_{x} \Phi\right)^{*}\right)^{-1}: \bigwedge_{j}^{\text {top }, 0} T_{x}^{*} M \rightarrow \bigwedge_{\Phi(j)}^{\text {top }, 0} T_{\Phi(x)}^{*} M .
$$

Then the isomorphisms $\Phi_{L}$ and $\varphi_{1}$ induce a linear isomorphism $\Phi_{*}$ from $\mathcal{H}_{k}$ to the space $\mathcal{H}_{k}^{\prime}$ consisting of the sections of $L^{k} \otimes \delta^{\prime}$ holomorphic with respect to $\Phi(j)$. Now suppose that

$$
S_{k}=T_{k} \circ \Phi_{*}: \mathcal{H}_{k} \rightarrow \mathcal{H}_{k}, \quad k=1,2, \ldots,
$$

for an endomorphism $T_{k}: \mathcal{H}_{k}^{\prime} \rightarrow \mathcal{H}_{k}$. Then comparing the definition of section 8.1 with the previous one, we prove that $\left(T_{k}\right)$ is a Fourier integral operator with symbol $\varphi_{2}$ in the sense of section 8.1 if and only if $\left(S_{k}\right)$ is a Fourier integral operator associated to $\Phi_{L}$ with symbol $\varphi=\varphi_{2} \circ \varphi_{1}$. This applies to the representation $R_{2}$ defined in Corollary 6.4. Indeed, for any $(A, e) \in \Gamma_{2}, R_{2}(A, e)$ is the composition of a pull-back with the map $\Psi_{A \tau, \tau}$, which is a Fourier integral operator by Theorem 8.2 .

Since the half-form bundle $\delta_{\tau}$ is the trivial bundle, we can identify its automorphisms with functions on $\mathrm{T}^{2}$, the correspondence being given by $\varphi_{x}\left(\Omega^{1 / 2}\right)=$ $f(x) \Omega^{1 / 2}$. We use this convention in the sequel for the symbols of the Fourier integral operators.

Theorem 9.1. For any $\tau$ and $(A, e) \in \Gamma_{2}$ the sequence

$$
R_{2}(A, e): H_{\tau}^{0}\left(\mathrm{~T}^{2}, L^{k} \otimes \delta\right) \rightarrow H_{\tau}^{0}\left(\mathrm{~T}^{2}, L^{k} \otimes \delta\right), \quad k=1,2, \ldots,
$$


is a Fourier integral operator associated to the prequantum lift of $A$ to L. Its symbol is the constant function equal to

$$
\sigma(A, e)=e(\tau)\left(\frac{A \tau-\overline{A \tau}}{\tau-\overline{A \tau}}\right)^{n / 2} .
$$

Applying Theorem 8.2 with $\tau_{1}=\tau_{2}$, the representation of the Weyl group $W$ is also given by Fourier integral operators.

Theorem 9.2. For any $\tau$ and $w \in W$ the sequence

$$
w: H_{\tau}^{0}\left(\mathrm{~T}^{2}, L^{k} \otimes \delta\right) \rightarrow H_{\tau}^{0}\left(\mathrm{~T}^{2}, L^{k} \otimes \delta\right), \quad k=1,2, \ldots,
$$

is a Fourier integral operator associated to the prequantum lift of $w$ to L. Its symbol is the constant function equal to 1.

9.2. Metaplectic group. Let $S$ be a symplectic vector space with a positive compatible complex structure $j$. Denote by $E=\operatorname{ker}(\mathrm{id}+i j)$ the space of vectors with type $(1,0)$. Let $\operatorname{Sp}(S)$ be the symplectic group of $S$. Using the complex structure we introduce a group $\operatorname{Mp}(S, j)$, isomorphic to the metaplectic group of $S . \operatorname{Mp}(S, j)$ consists of the pairs $(A, z)$ such that $A \in \mathrm{Sp}(S)$ and $z$ is a complex number satisfying

$$
z^{2}=\operatorname{det}\left(g^{-1} \pi_{E, g E}: E \rightarrow E\right) .
$$

Here $\pi_{E, g E}$ is the projection from $E$ onto $g E$ with kernel $\bar{E}$. The product of $\operatorname{Mp}(S, j)$ is determined by the condition that the projection onto the symplectic group is a group morphism and that the identity is the pair (id,1). We shall also consider an extension $\mathrm{Mp}_{2}(S, j)$ by $\mathbb{Z}_{4}=\{ \pm 1, \pm i\}$ of the symplectic group. It is defined as the set of pairs $(A, z)$ such that $z^{4}=\operatorname{det}^{2}\left(g^{-1} \pi_{E, g E}: E \rightarrow E\right)$. The product is determined by the condition that the map

$$
\operatorname{Mp}(S, j) \times \mathbb{Z}_{4} \rightarrow \operatorname{Mp}_{2}(S, j)
$$

sending $(A, z, u)$ into $(A, z u)$ is a group morphism.

We apply these constructions to $S=\mathfrak{t} \oplus \mathfrak{t}=\mathbb{R}^{2} \otimes \mathfrak{t}$ with the complex structure given by some $\tau$ in the upper half-plane. Then the symbols of the operators defining the representation $R_{2}$ belongs to the metaplectic group.

Proposition 9.3. We have a group morphism from $\Gamma_{2}$ to $\mathrm{Mp}\left(\mathfrak{t} \oplus \mathfrak{t}, j_{\tau}\right)$ sending $(A, e)$ into $\left(A \otimes \operatorname{id}_{\mathfrak{t}}, \sigma(A, e)\right)$ with

$$
\sigma(A, e)=e(\tau)\left(\frac{A \tau-\overline{A \tau}}{\tau-\overline{A \tau}}\right)^{n / 2} .
$$

Proof. Observe that for any $g \in \operatorname{Sp}(S)$, the endomorphism

$$
\pi_{E, g E}^{*} \circ\left(g^{-1}\right)^{*}: \bigwedge^{\text {top }} E^{*} \rightarrow \bigwedge_{\text {top }}(g E)^{*} \rightarrow \bigwedge^{\text {top }} E^{*}
$$

is the multiplication by $\operatorname{det}\left(g^{-1} \pi_{E, g E}: E \rightarrow E\right)$.

Let us apply this to $g=A \otimes \mathrm{id}_{\mathfrak{t}}$. By equation (27) and the condition $e(\tau)^{2}=$ $(-c \tau+d)^{n}$, the pull-back by $g^{-1}$ is multiplication by $e^{2}(\tau)$. By Lemma 8.1, $\pi_{E, g E}^{*}$ is the multiplication by $\left(\frac{A \tau-\overline{A \tau}}{\tau-\overline{A \tau}}\right)^{n}$. This implies that $\left(A \otimes \mathrm{id}_{\mathfrak{t}}, \sigma(A, e)\right)$ belongs to the metaplectic group $\operatorname{Mp}\left(\mathfrak{t} \oplus \mathfrak{t}, j_{\tau}\right)$.

One shows that the map is a group morphism by extending it to the group defined as $\Gamma_{2}$ by replacing $\operatorname{Sl}(2, \mathbb{Z})$ with $\operatorname{Sl}(2, \mathbb{R})$ and using a continuity argument. 
For any $A \in \operatorname{Sl}(2, \mathbb{R})$, let $d(A, \tau)=\operatorname{det}\left(A^{-1} \pi_{E, A E}: E \rightarrow E\right)$ with $E$ the complex polarization determined by the complex structure $p+\tau q$. Then in the proof of the previous proposition we showed that

$$
d(A, \tau)=(-c \tau+d) \frac{A \tau-\overline{A \tau}}{\tau-\overline{A \tau}}
$$

We will use this equation several times in the sequel. Assume that the rank of $G$ is even, so $n=2 p$. We have a morphism from $\Gamma$ into $\Gamma_{2}$ sending $A$ into $\left(A,(-c \tau+d)^{p}\right)$. Composed with the morphism provided by Proposition 9.3 , we obtain the group morphism

$$
\Gamma \rightarrow \operatorname{Mp}\left(\mathfrak{t} \oplus \mathfrak{t}, j_{\tau}\right), \quad A \rightarrow\left(A \otimes \mathrm{id}_{\mathfrak{t}}, d(A, \tau)^{p}\right) .
$$

Now assume that the rank of $G$ is odd, $n=2 p+1$. Introduce the subgroup $\operatorname{Mp}(\mathbb{Z}, \tau)$ of $\operatorname{Mp}\left(\mathbb{R}^{2}, \tau\right)$ consisting of the pairs $(A, z) \in \Gamma \times \mathbb{C}^{*}$ such that $z^{2}=d(A, \tau)$. Using (46) again, we prove that this group is isomorphic to $\Gamma_{2}$, the isomorphism being given by

$$
(A, z) \in \operatorname{Mp}(\mathbb{Z}, \tau) \rightarrow\left(A, z(-c \tau+d)^{p}\left(\frac{A \tau-\overline{A \tau}}{\tau-\overline{A \tau}}\right)^{-1 / 2}\right) \in \Gamma_{2}
$$

Finally, composing this morphism with the one of Proposition 9.3, we obtain the group morphism

$$
\operatorname{Mp}(\mathbb{Z}, \tau) \rightarrow \operatorname{Mp}\left(\mathfrak{t} \oplus \mathfrak{t}, j_{\tau}\right), \quad(A, z) \rightarrow\left(A \otimes \mathrm{id}_{\mathfrak{t}}, z d(A, \tau)^{p}\right) .
$$

Considering the representation of the Weyl group, we obtain a morphism into the extension $\mathrm{Mp}_{2}\left(\mathfrak{t} \oplus \mathfrak{t}, j_{\tau}\right)$ of the symplectic group.

Proposition 9.4. We have a group morphism from $W$ to $\mathrm{Mp}_{2}\left(\mathfrak{t} \oplus \mathfrak{t}, j_{\tau}\right)$ sending $w$ into $\left(\operatorname{id}_{\mathbb{R}^{2}} \otimes w, 1\right)$.

More generally, if $w$ is an element of the orthogonal group of $\mathfrak{t}$, then $\left(\operatorname{id}_{\mathbb{R}^{2}} \otimes w, u\right)$ belongs to the metaplectic group (resp. the extension by $\mathbb{Z}_{4}$ ) if and only if $u^{2}=$ $\operatorname{det} w\left(\right.$ resp. $\left.u^{4}=1\right)$.

9.3. Index computation. As previously, consider the metaplectic group $\operatorname{Mp}(S, j)$ of a symplectic vector space endowed with a complex structure. Let $\operatorname{Mp}_{*}(S, j)$ be the subset consisting of the pairs $(g, z)$ such that 1 is not an eigenvalue of $g$. Then we defined in 3 an index map

$$
\text { ind }: \operatorname{Mp}_{*}(S, j) \rightarrow \mathbb{Z} / 4 \mathbb{Z} \text {. }
$$

It is continuous and takes distinct values on each of the four components of $\mathrm{Mp}_{*}(S, j)$. To compute it, we only need the two following properties. If $E$ has dimension 2, then

$$
\operatorname{ind}(g, z)=k+\frac{1}{2}\left(1-(-1)^{k+\epsilon}\right),
$$

where $k \in \mathbb{Z}$ is such that the argument of $z$ belongs to $\left[\frac{\pi}{2} k, \frac{\pi}{2}(k+1)\right.$ [ and $\epsilon$ is equal to 0 if the trace of $g$ is larger than 2 and to 1 otherwise. Furthermore, if $\left(g_{1}, z_{1}\right) \in \operatorname{Mp}_{*}\left(S_{1}, j_{1}\right)$ and $\left(g_{2}, z_{2}\right) \in \operatorname{Mp}_{*}\left(S_{2}, j_{2}\right)$, then $\left(g_{1} \oplus g_{2}, z_{1} z_{2}\right)$ belongs to $\operatorname{Mp}_{*}\left(S_{1} \oplus S_{2}, j_{1} \oplus j_{2}\right)$ and

$$
\operatorname{ind}\left(g_{1} \oplus g_{2}, z_{1} z_{2}\right)=\operatorname{ind}\left(g_{1}, z_{1}\right)+\operatorname{ind}\left(g_{2}, z_{2}\right) .
$$

The elements $(g, z)$ of $\mathrm{Mp}_{2}(S, j)$ such that 1 is not an eigenvalue of $g$ also have an index defined modulo $4 \mathbb{Z}$. It is such that

$$
\operatorname{ind}\left(g, i^{k} z\right)=k+\operatorname{ind}(g, z)
$$


if $(g, z) \in \mathrm{Mp}_{*}(S, j)$. In the following we compute the index of some elements of the metaplectic group of $S=\mathfrak{t} \oplus \mathfrak{t}$ endowed with the complex structure determined by $\tau \in \mathcal{H}_{+}$.

Lemma 9.5. For any hyperbolic $A \in \mathrm{Sl}(2, \mathbb{R})$ and $w$ in the orthogonal group of $\mathfrak{t}$, we have

$$
\operatorname{ind}(A \otimes w, z)=\operatorname{ind}\left(A \otimes \operatorname{id}_{\mathfrak{t}}, z\right),
$$

where $z$ is any complex number such that $\left(A \otimes \mathrm{id}_{\mathfrak{t}}, z\right)$ belongs to $\mathrm{Mp}_{2}\left(\mathfrak{t} \oplus \mathfrak{t}, j_{\tau}\right)$.

Proof. $A$ being hyperbolic, 1 is not an eigenvalue of $A \otimes w$. Since

$$
\left(A \otimes \operatorname{id}_{\mathfrak{t}}, z\right) \cdot\left(\operatorname{id}_{\mathbb{R}^{2}} \otimes w, u\right)=(A \otimes w, z u),
$$

the fact that $\left(A \otimes \mathrm{id}_{\mathfrak{t}}, z\right)$ belongs to $\mathrm{Mp}_{2}\left(\mathfrak{t} \oplus \mathfrak{t}, j_{\tau}\right)$ implies that $(A \otimes w, z)$ also belongs to $\mathrm{Mp}_{2}\left(\mathfrak{t} \oplus \mathfrak{t}, j_{\tau}\right)$. Let us prove that they have the same index. Since the index is locally constant, the result is straightforward if $w$ belongs to the special orthogonal group. Otherwise we may assume that $w$ is a reflexion and that $A$ is the diagonal matrix with coefficient $2,1 / 2$. Let us decompose $\mathfrak{t}$ as a direct sum of orthogonal lines. The complex structure $j_{\tau}$ preserves the associated decomposition of $\mathbb{R}^{2} \otimes \mathfrak{t}$. So using (51) it is sufficient to prove that

$$
\operatorname{ind}(A, u)=\operatorname{ind}(-A, u) \text {. }
$$

One may assume that $(A, u) \in \mathrm{Mp}\left(\mathbb{R}^{2}, \tau\right)$ so that $(-A, i u) \in \mathrm{Mp}\left(\mathbb{R}^{2}, \tau\right)$. Then the result follows from formula (50).

We can give explicit formulas for the index of $\left(A \otimes \mathrm{id}_{\mathfrak{t}}, z\right)$ by decomposing $\mathbb{R}^{2} \otimes \mathfrak{t}$ into a direct sum of $\mathbb{R}^{2}$ 's as we did in the previous proof.

Lemma 9.6. If $n=2 p$, for any $A \in \mathrm{Sl}_{*}(2, \mathbb{R})$, we have

$$
\operatorname{ind}\left(A \otimes \operatorname{id}_{\mathfrak{t}}, d(A)^{p}\right)=2 \epsilon p,
$$

where $\epsilon$ is equal to 0 if the trace of $A$ is larger than 2 and to 1 otherwise. If $n=2 p+1$, for any $(A, z) \in \mathrm{Mp}_{*}\left(\mathbb{R}^{2}, \tau\right)$, we have

$$
\operatorname{ind}\left(A \otimes \operatorname{id}_{\mathfrak{t}}, d(A)^{p} z\right)=2 \epsilon p+\operatorname{ind}(A, z),
$$

where $\epsilon$ is defined as previously.

In the second case, the index of $(A, z)$ is given by (50). With these formulas we obtain the index of any element in the images of the morphisms (47) and (49).

Proof. Working with the decomposition of $\mathbb{R}^{2} \otimes \mathfrak{t}$, we only have to consider $n=2$. We have

$$
\operatorname{ind}(A \oplus A, d(A))=2 \operatorname{ind}(A, z),
$$

where $z^{2}=d(A)$. We conclude with formula (50).

9.4. Trace estimates. Under a transversality condition, the trace of a Fourier integral operator admits an asymptotic expansion and we can explicitly compute the leading term in terms of the symbol. The next theorem has been proved in 3 . We restrict ourselves to the case of $\mathrm{T}^{2}$ to simplify the statement. 
Theorem 9.7. Let $\Phi$ be a symplectomorphism of $\mathrm{T}^{2}$ whose graph transversally intersects the diagonal. Let $\Phi_{L}$ be a prequantum bundle isomorphism of $L$ lifting $\Phi$ and $\left(T_{k}: H_{\tau}^{0}\left(\mathrm{~T}^{2}, L^{k} \otimes \delta_{\tau}\right) \rightarrow H_{\tau}^{0}\left(\mathrm{~T}^{2}, L^{k} \otimes \delta_{\tau}\right)\right)$ be a Fourier integral operator associated to $\Phi_{L}$ with symbol $f$. Then for any fixed point $x$ of $\Phi$, there exists a sequence $\left(a_{\ell, x}\right)$ of complex numbers such that for any $N$,

$$
\operatorname{tr}\left(T_{k}\right)=\sum_{x / \Phi(x)=x} u_{x}^{k}\left(a_{x, 0}+a_{x, 1} k^{-1}+\ldots+a_{x, N} k^{-N}+O\left(k^{-N-1}\right)\right),
$$

where for any $x, u_{x}$ is the trace of $\Phi_{L}(x): L_{x} \rightarrow L_{x}$. Furthermore, if $\left(T_{x} \Phi, f(x)\right)$ is an element of $\mathrm{Mp}_{2}\left(\mathfrak{t} \oplus \mathfrak{t}, j_{\tau}\right)$, then

$$
a_{x, 0}=\frac{i^{\operatorname{ind}\left(T_{x} \Phi, f(x)\right)}}{\left|\operatorname{det}\left(\operatorname{id}-T_{x} \Phi\right)\right|^{1 / 2}} .
$$

We apply this to estimate the character of the representation $R_{2}^{\text {alt }}$. First we have

$$
\operatorname{tr}\left(R_{2}^{\text {alt }}(A, e)\right)=\frac{1}{|W|} \sum_{w \in W}(-1)^{\ell(w)} \operatorname{tr}\left(w \cdot R_{2}(A, e)\right) .
$$

Then by Theorems 9.1 and $9.2 w \cdot R_{2}(A, e)$ is a Fourier integral operator associated to the prequantum lift $A \otimes w$. Its symbol is the constant map equal to $\sigma(A, e)$. By Lemma 9.5 the index of $(A \otimes w, \sigma(A, e))$ doesn't depend on $w$. We easily compute the action of the prequantum lift of $A \otimes w$ at the fixed points and obtain the following theorem.

Theorem 9.8. For any $(A, e) \in \Gamma_{2}$ such that $A$ is hyperbolic, we have

$$
\operatorname{tr}\left(R_{2}^{\text {alt }}(A, e)\right) \sim \frac{i^{n(A, e)}}{|W|} \sum_{\substack{w \in W \\ u \in \mathrm{T}^{2} /(A \otimes w) . x=x}}(-1)^{\ell(w)} \frac{e^{i k \theta(A \otimes w, x)}}{|\operatorname{det}(\mathrm{id}-A \otimes w)|^{1 / 2}},
$$

where

- $n(A, e)$ is the index of $\left(A \otimes \mathrm{id}_{\mathfrak{t}}, \sigma(A, e)\right)$,

- $\theta(A \otimes w, x)=\pi(B(\mu, p)-B(\gamma, q)+B(\gamma, \mu))$ if $x$ is the class of $(p, q) \in \mathfrak{t}^{2}$ and $(\gamma, \mu)=(A \otimes w)(p, q)-(p, q)$.

We can explicitly compute the indices with Lemma 9.6. For the statement in the introduction we used the two morphisms (47) and (49).

\section{Appendix A. Proofs of Theorems 5.1 and 5.2}

A.1. The basis of $H_{\tau}^{0}\left(T^{2}, L^{k}\right)$. Recall that the holomorphic sections of $L^{k}$ identify with the sections over $\mathfrak{t}^{2}$ of the form $f s^{k}$ such that $f: \mathfrak{t}^{2} \rightarrow \mathbb{C}$ is holomorphic and $f s^{k}$ is $\Lambda^{2}$-invariant. As shown in a straightforward computation from (18) and (6), this invariance is equivalent to

$$
f(p+\dot{p}, q+\dot{q})=f(p, q) \exp \left(-2 i \pi k\left(B(\zeta, \dot{q})+\frac{\tau}{2} B(\dot{q}, \dot{q})\right)\right)
$$

for all $\dot{p}, \dot{q}$ in $\Lambda$. Then to prove that the sections $\Theta_{\mu, k} s^{k}$ form a basis of the holomorphic sections of $L^{k}$, we decompose the functions $f$ as a Fourier series in the $p$ variable with coefficients depending on $q$. Then the holomorphy and the equivariance in the $q$-directions translate into a condition on the coefficients, leading to the result. 
Let us show that the sections $\Theta_{\mu, k} s^{k}$ are mutually orthogonal and compute their norms. Let $m$ be the Riemannian volume of $\mathfrak{t}$. The Liouville measure $\left|\omega^{n}\right| / n$ ! of $\mathfrak{t}^{2}$ is equal to $(2 \pi)^{n} m(p) \otimes m(q)$. The scalar product of $f s^{k}$ and $f^{\prime} s^{k}$ is given by

$$
(2 \pi)^{n} \int_{(p, q) \in D^{2}} f(p, q) \overline{f^{\prime}(p, q)}|s(p, q)|^{2 k} m(p) \otimes m(q) .
$$

Here $D$ is the fundamental domain $\left\{x_{1} \mu_{1}+\ldots+x_{n} \mu_{n} /\left(x_{i}\right) \in[0,1]^{n}\right\}$, where $\left(\mu_{i}\right)$ is a basis of the lattice $\Lambda$. Now a straightforward computation starting from (19) shows that

$$
\Theta_{\mu, k} \bar{\Theta}_{\mu^{\prime}, k}|s(p, q)|^{2 k}=\sum_{\substack{\gamma \in \mu+\Lambda \\ \gamma^{\prime} \in \mu^{\prime}+\Lambda}} c_{\gamma, \gamma^{\prime}}(q) \exp \left(2 i \pi k B\left(p, \gamma^{\prime}-\gamma\right)\right),
$$

where the diagonal coefficients are given by

$$
c_{\gamma, \gamma}(q)=\exp (i \pi k(\tau-\bar{\tau}) B(q-\gamma, q-\gamma)) .
$$

Integrating with respect to $p$, one deduces that the scalar product of $\Theta_{\mu, k}$ and $\Theta_{\mu^{\prime}, k}$ vanishes when $\mu \neq \mu^{\prime} \bmod \Lambda$. Furthermore,

$$
\left\|\Theta_{\mu, k} s^{k}\right\|^{2}=(2 \pi)^{n} \operatorname{Vol}(\mathfrak{t} / \Lambda) \sum_{\gamma \in \mu+\Lambda} \int_{D} c_{\gamma, \gamma}(q) m(q) .
$$

Using that $t$ is the disjoint union of the $-\gamma+D$ when $\gamma$ runs over $\mu+\Lambda$, we obtain

$$
\begin{aligned}
\sum_{\gamma \in \mu+\Lambda} \int_{D} c_{\gamma, \gamma}(q) m(q) & =\int_{\mathfrak{t}} \exp (i \pi k(\tau-\bar{\tau}) B(q, q)) m(q) \\
& =\left(\frac{i}{k(\tau-\bar{\tau})}\right)^{n / 2}
\end{aligned}
$$

which ends the computation of the norm.

A.2. The action of $S$ and $T$ in the basis of theta functions. Let us prove Theorem 5.2. Denote by $\varphi_{A}$ the map sending $(p, q)$ into $(a p+b q, c p+d q)$. Recall that $\zeta_{\tau}=p+\tau q$ and $s_{\tau}=\exp \left(i \pi B\left(\zeta_{\tau}, q\right)\right)$. Then

so

$$
\varphi_{A}^{*} \zeta_{A \cdot \tau}=\frac{\zeta_{\tau}}{-c \tau+d}, \quad \varphi_{A}^{*} s_{A \cdot \tau}=\exp \left(i \pi \frac{c B\left(\zeta_{\tau}, \zeta_{\tau}\right)}{-c \tau+d}\right) s_{\tau},
$$

$$
\varphi_{A}^{*}\left(f\left(\zeta_{A . \tau}\right) s_{A . \tau}^{k}\right)=f\left(\frac{\zeta_{\tau}}{-c \tau+d}\right) \exp \left(i \pi k \frac{c B\left(\zeta_{\tau}, \zeta_{\tau}\right)}{-c \tau+d}\right) s_{\tau}^{k} .
$$

In particular, for $A=T^{-1}$, this gives

$$
\varphi_{A}^{*}\left(f\left(\zeta_{A . \tau}\right) s_{A . \tau}^{k}\right)=f\left(\zeta_{\tau}\right) s_{\tau}^{k}
$$

so that

$$
\varphi_{A}^{*}\left(\Theta_{\mu, k}^{A . \tau} s_{A . \tau}^{k}\right)=\sum_{\gamma \in \mu+\Lambda} \exp \left(2 i \pi k\left(\frac{\tau+1}{2} B(\gamma, \gamma)-B\left(\zeta_{\tau}, \gamma\right)\right)\right) s_{\tau}^{k} .
$$

Using that $B$ takes integral even values on the diagonal of $\Lambda^{2}$, one shows that $\exp (i k \pi B(\gamma, \gamma))=\exp (i k \pi B(\mu, \mu))$ for any $\gamma \in \mu+\Lambda$. This implies that

$$
\varphi_{A}^{*}\left(\Theta_{\mu, k}^{A . \tau} s_{A . \tau}^{k}\right)=\sum_{\gamma \in \mu+\Lambda} \exp (i k \pi B(\mu, \mu)) \Theta_{\mu, k}^{\tau} s_{\tau}^{k},
$$

which proves the second formula of the theorem. 
Now assume that $A=S^{-1}$. Then

$$
\varphi^{*}\left(f\left(\zeta_{A \cdot \tau}\right) s_{A \cdot \tau}^{k}\right)=f\left(\frac{\zeta_{\tau}}{\tau}\right) \exp \left(-\frac{i \pi k}{\tau} B\left(\zeta_{\tau}, \zeta_{\tau}\right)\right) s_{\tau}^{k}
$$

Applying to the theta functions, we have

$$
\varphi^{*}\left(\Theta_{\mu, k}^{A \cdot \tau} s_{A \cdot \tau}^{k}\right)=\sum_{\gamma \in \mu+\Lambda} \exp \left(-\frac{i \pi k}{\tau} B\left(\gamma+\zeta_{\tau}, \gamma+\zeta_{\tau}\right)\right) s_{\tau}^{k} .
$$

Applying the Poisson summation formula, we obtain after some computations that $\varphi^{*}\left(\Theta_{\mu, k}^{A . \tau} s_{A . \tau}^{k}\right)$ is equal to

$$
\left(\frac{\tau}{i k}\right)^{n / 2} \operatorname{Vol}(\mathfrak{t} / \Lambda)^{-1} \sum_{\gamma \in k^{-1} \Lambda^{*}} \exp \left(2 i \pi k\left(\frac{\tau}{2} B(\gamma, \gamma)-B\left(\mu+\zeta_{\tau}, \gamma\right)\right)\right) s_{\tau}^{k}
$$

Since $k^{-1} \Lambda^{*}=\bigcup\left(\mu^{\prime}+\Lambda\right)$, where $\mu^{\prime}$ runs over $k^{-1} \Lambda^{*} \bmod \Lambda$, this is equal to

$$
\left(\frac{\tau}{i k}\right)^{n / 2} \operatorname{Vol}(\mathfrak{t} / \Lambda)^{-1} \sum_{\substack{\mu^{\prime} \in k^{-1} \Lambda^{*} \\ \bmod \Lambda}} \exp \left(-2 i \pi k B\left(\mu, \mu^{\prime}\right)\right) \Theta_{\mu^{\prime}, k}^{\tau} s_{\tau}^{k},
$$

which completes the proof.

\section{Appendix B. Index}

Lie group notation:

$\mathfrak{g}, B \quad$ Lie algebra of $G$ and its basic inner product; 2

$\mathrm{T}, \mathfrak{t}, \Lambda \quad$ maximal torus, its Lie algebra and integral lattice; 2

$\mathfrak{A}, W \quad$ open fundamental Weyl alcove and Weyl group; 2

$\ell: W \rightarrow\{ \pm 1\} \quad$ alternating character; 2

Moduli space and its quantization:

$p, q, \zeta=p+\tau q \quad$ projections from $\mathfrak{t}^{2}$ onto $\mathfrak{t}$, complex coordinates; $3.1,5.1$

$\omega, L_{\mathfrak{t}^{2}} \quad$ symplectic form and prequantum bundle of $\mathfrak{t}^{2} ; 3.1$

$L \quad$ prequantum bundle of $\mathrm{T}^{2} ; 3.2$

$s, \Theta_{\mu, k} \quad$ section of $L_{\mathfrak{t}^{2}}$ and theta function; 5.1

$\left(\chi_{\mu, k}\right)_{\mu} \quad$ basis of alternating sections of $H_{\tau}^{0}\left(\mathrm{~T}^{2}, L^{k}\right) ; 5.2$

Modular group extensions and their representation:

$\Gamma_{2}, R_{2} \quad$ extension by $\mathbb{Z}_{2}$ of $\Gamma$, representation in $H_{\tau}^{0}\left(\mathrm{~T}^{2}, L^{k}\right) ; 6.2$

$R_{2}^{\text {alt }} \quad$ representation in the subspace of alternating sections; 6.2

$\Gamma_{\infty}, R_{\infty}$ extension by $\mathbb{Z}$ of $\Gamma$ and its representation; 7.1

$\Gamma_{4}, R_{4} \quad$ extension by $\mathbb{Z}_{4}$ of $\Gamma$ and its representation; 7.2

\section{REFERENCES}

[1] Bojko Bakalov and Alexander Kirillov, Jr. Lectures on tensor categories and modular functors, volume 21 of University Lecture Series. American Mathematical Society, Providence, RI, 2001. MR1797619 (2002d:18003)

[2] L. Charles. Quasimodes and Bohr-Sommerfeld conditions for the Toeplitz operators. Comm. Partial Differential Equations, 28(9-10):1527-1566, 2003. MR2001172 (2005d:53142)

[3] L. Charles. A Lefschetz fixed point formula for symplectomorphisms. Journal of Geometry and Physics, 60:1890-1902, December 2010. MR2735276

[4] L. Charles. Asymptotic properties of the quantum representations of the mapping class group, 2010, arXiv:1005.3452.

[5] Daniel S. Freed. Remarks on Chern-Simons theory. Bull. Amer. Math. Soc. (N.S.), 46(2):221254, 2009. MR2476413 (2010a:58031) 
[6] Doron Gepner and Edward Witten. String theory on group manifolds. Nuclear Phys. B, 278(3):493-549, 1986. MR862896 (88h:81169)

[7] Lisa C. Jeffrey. Chern-Simons-Witten invariants of lens spaces and torus bundles, and the semiclassical approximation. Comm. Math. Phys., 147(3):563-604, 1992. MR1175494 (93f:57042)

[8] Victor G. Kac and Dale H. Peterson. Infinite-dimensional Lie algebras, theta functions and modular forms. Adv. in Math., 53(2):125-264, 1984. MR750341 (86a:17007)

[9] Alexander A. Kirillov, Jr. On an inner product in modular tensor categories. J. Amer. Math. Soc., 9(4):1135-1169, 1996. MR 1358983 (97f:18007)

[10] David Mumford. Tata lectures on theta. III, volume 97 of Progress in Mathematics. Birkhäuser Boston Inc., Boston, MA, 1991. With the collaboration of Madhav Nori and Peter Norman. MR2307768 (2007k:14087)

[11] N. Reshetikhin and V. G. Turaev. Invariants of 3-manifolds via link polynomials and quantum groups. Invent. Math., 103(3):547-597, 1991. MR1091619(92b:57024)

[12] V. G. Turaev. Quantum invariants of knots and 3-manifolds, volume 18 of de Gruyter Studies in Mathematics. Walter de Gruyter \& Co., Berlin, 1994. MR.1292673 (95k:57014)

[13] Edward Witten. Quantum field theory and the Jones polynomial. Comm. Math. Phys., 121(3):351-399, 1989. MR990772 (90h:57009)

Institut de Mathématiques de Jussieu (UMR 7586), Université Pierre et Marie Curie - Paris 6, Paris, F-75005 France 